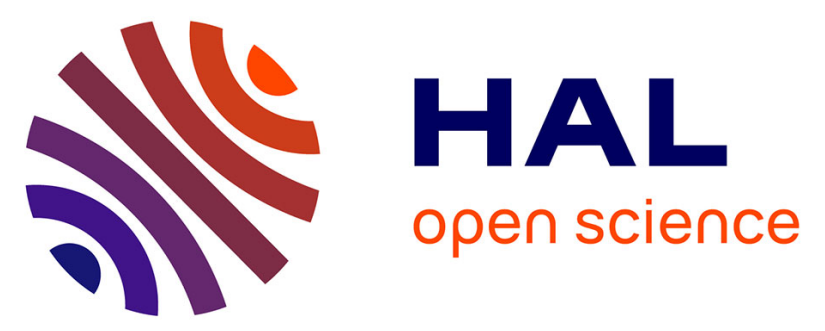

\title{
Empirical comparison of two methods for the Bayesian update of the parameters of probability distributions in a two-level hybrid probabilistic-possibilistic uncertainty framework for risk assessment
}

Nicola Pedroni, Enrico Zio, A Pasanisi, M Couplet

\section{To cite this version:}

Nicola Pedroni, Enrico Zio, A Pasanisi, M Couplet. Empirical comparison of two methods for the Bayesian update of the parameters of probability distributions in a two-level hybrid probabilisticpossibilistic uncertainty framework for risk assessment. ASCE-ASME Journal of Risk and Uncertainty in Engineering Systems, Part A: Civil Engineering, 2015, pp.04015015. 10.1061/AJRUA6.0000848 . hal-01270598

\section{HAL Id: hal-01270598 \\ https://hal.science/hal-01270598}

Submitted on 10 Feb 2016

HAL is a multi-disciplinary open access archive for the deposit and dissemination of scientific research documents, whether they are published or not. The documents may come from teaching and research institutions in France or abroad, or from public or private research centers.
L'archive ouverte pluridisciplinaire HAL, est destinée au dépôt et à la diffusion de documents scientifiques de niveau recherche, publiés ou non, émanant des établissements d'enseignement et de recherche français ou étrangers, des laboratoires publics ou privés. 


\title{
1 Empirical comparison of two methods for the Bayesian update
}

\section{of the parameters of probability distributions in a two-level}

\section{hybrid probabilistic-possibilistic uncertainty framework for}

\section{risk assessment}

\author{
N. Pedroni ${ }^{1}$, E. Zio $^{2}$, A. Pasanisi ${ }^{3}$, M. Couplet $^{4}$
}

\begin{abstract}
In this paper, we address the issue of updating in a Bayesian framework, the possibilistic representation of the epistemically-uncertain parameters of (aleatory) probability distributions, as new information (e.g., data) becomes available. Two approaches are considered: the first is based on a purely possibilistic counterpart of the classical, well-grounded probabilistic Bayes' theorem; the second relies on the hybrid combination of (i) Fuzzy Interval Analysis (FIA) to process the uncertainty described by possibility distributions and (ii) repeated Bayesian updating of the uncertainty represented by probability distributions.
\end{abstract}

The feasibility of the two methods is shown on a literature case study involving the risk-based design of a flood protection dike.

Keywords: hierarchical two-level uncertainty, Bayesian update, possibility distributions, fuzzy interval analysis, possibilistic Bayes theorem, flood protection dike.

\footnotetext{
${ }^{1}$ Corresponding author. Assistant professor at the Chair of system science and the energetic challenge-Fondation EdF at CentraleSupélec, 3 Rue Joliot Curie - Plateau de Moulon, 91190, Gif-Sur-Yvette, France. E-mail: nicola.pedroni@ supelec.fr.

${ }^{2}$ Full professor at the Chair of system science and the energetic challenge-Fondation EdF at CentraleSupélec, Grande Voie des Vignes, 92290, Chatenay-Malabry, France. E-mail: enrico.zio@ecp.fr. Also: Energy Department, Politecnico di Milano, Via Ponzio, 34/3 - 20133 Milano, Italy. E-mail: enrico.zio@polimi.it.

${ }^{3}$ Project Manager at EDF R\&D - EIFER, Emmy-Noether-Str. 11. 76131 Karlsruhe, Germany. E-mail address: alberto.pasanisi@edf.fr.

${ }^{4}$ Research Engineer at Electricité de France, R\&D, 6 Quai Watier, 78400, Chatou, France. E-mail address: mathieu.couplet@edf.fr.
} 
 \\ Introduction}

23 We consider a framework of uncertainty representation with two hierarchical levels [Limbourg and contain parameters (e.g., probabilities, failure rates, ...) that are epistemically-uncertain, i.e., known with poor precision due to lack of knowledge and information. Traditionally, both types of uncertainty are represented by probability distributions [Apostolakis and Kaplan, 1981; Apostolakis, 1990; NUREG-CR-6850, 2005; USNRC, 2002 and 2009; NASA, 2010] and Bayes' rule is useful for updating the (probabilistic) epistemic uncertainty representation as new information (e.g., data) becomes available [Bernardo and Smith, 1994; Siu and Kelly, 1998;

31 Lindley, 2000 and 2006; Bedford and Cooke, 2001; Atwood et al., 2003; Kelly and Smith, 2009 and 2011; Pasanisi et al., 2012].

However, in some situations, insufficient knowledge, information and data impair a probabilistic representation of epistemic uncertainty. A number of alternative representation frameworks have been proposed for such cases [Aven, 2010 and 2011; Aven and Steen, 2010; Aven and Zio, 2011; theory [Molchanov, 2005], evidence theory [Ferson et al., 2003 and 2004; Helton et al., 2007 and 2008; Sentz and Ferson, 2002; Le Duy et al., 2013; Sallak et al., 2013], possibility theory (that can be considered a special case of evidence theory) [Baudrit and Dubois, 2006; Baudrit et al., 2006 and 2008; Dubois, 2006; Dubois and Prade, 1988], probability bound analysis using probability boxes (p-boxes) [Ferson and Ginzburg, 1996; Crespo et al., 2013; Mehl, 2013], interval analysis [Ferson and Hajagos, 2004; Ferson and Tucker, 2006; Ferson et al., 2007 and 2010; Jalal-Kamali and Kreinovich, 2013; Muscolino and Sofi, 2013; Zhang et al., 2013] and interval probabilities [Weichselberger, 2000]; notice that most of these theories can be included within the general common framework of imprecise probabilities [Kuznetsov, 1991; Walley, 1991; Kozine and 
Filimonov, 2000; Kozine and Utkin, 2002; Coolen and Utkin, 2007; Beer and Ferson, 2013; Beer et al., 2013a; Blockley, 2013; Reid, 2013; Sankararaman and Mahadevan, 2013].

In this paper, we adopt possibility distributions to describe epistemic uncertainty and address the issue of updating, in a Bayesian framework, the possibilistic representation of the epistemicallyuncertain parameters of (aleatory) probability distributions. We take two approaches of literature. The first is based on a purely possibilistic counterpart of the classical, well-grounded probabilistic Bayes' theorem: it requires the construction of a possibilistic likelihood function which is used to revise the prior possibility distributions of the uncertain parameters (determined on the basis of a priori subjective knowledge and/or data) [Dubois and Prade, 1997; Lapointe and Bobee, 2000]. This approach has been already applied by the authors for updating possibility distributions in [Pedroni et al., 2014]. The second is a hybrid probabilistic-possibilistic method that relies on the use of Fuzzy Probability Density Functions (FPDFs), i.e., PDFs with possibilistic (fuzzy) parameters. It is based on the combination of (i) Fuzzy Interval Analysis (FIA) to process the uncertainty described by possibility distributions and (ii) repeated Bayesian updating of the uncertainty represented by probability distributions [Beer, 2009a; Stein and Beer, 2011; Stein et al., 2013; Beer et al., 2014a].

The objective (and the main contribution of the paper) is to compare the effectiveness of the two methods. To the best of the authors' knowledge, this is the first time that the above mentioned techniques are systematically compared with reference to risk assessment problems where hybrid uncertainty is separated into two hierarchical levels. To keep the analysis simple and retain a clear view of each step, the investigations are carried out with respect to a simple literature case study involving the risk-based design of a flood protection dike [Pasanisi et al., 2009; Limbourg and de Rocquigny, 2010]. In addition, different numerical indicators (e.g., cumulative distributions, exceedance probabilities, percentiles, ...) are considered to perform a fair, quantitative comparison between the methods and evaluate their rationale and appropriateness in relation to risk analysis.

Other methods have been proposed in the literature to revise, in a Bayesian framework, nonprobabilistic representations of epistemic uncertainty [Ferson, 2005]. In [Viertl, 1996, 1997, 1999, 
2008a,b and 2011; Viertl and Hareter, 2004a,b; Viertl and Hule, 1991] a modification of the Bayes' theorem is presented to account for the presence of fuzzy data and fuzzy prior PDFs: the approach is similar to that employed by [Beer, 2009a; Stein and Beer, 2011; Stein et al., 2013] and considered in this paper. In [Smets, 1993] a Generalized Bayes Theorem (GBT) is proposed within the framework of evidence theory: this approach is applied by [Le-Duy et al., 2011] to update the estimates of the failure rates of mechanical components in the context of nuclear Probabilistic Risk Assessment (PRA). Finally, in [Walley, 1996; Bernard, 2005; Masegosa and Moral, 2014] Imprecise Dirichlet Models (IDMs) are proposed for objective statistical inference from multinomial data. In the IDM, prior or posterior uncertainty about a parameter is described by a set of Dirichlet distributions, and inferences about events are summarized by lower and upper probabilities. This model has been extended by [Quaeghebeur and de Cooman, 2005] to generalized Bayesian inference from canonical exponential families and by [Walter and Augustin, 2009] with the aim of handling prior-data conflicts.

The remainder of the paper is organized as follows. First, the representation of aleatory (probabilistic) and epistemic (possibilistic) uncertainties in a "two-level" framework is provided; then, the two methods employed in this paper for the Bayesian update of the possibilistic parameters of aleatory probability distributions are described in details; after that, the case study concerning the risk-based design of a flood protection dike is presented; in the following Section, the methods described are applied to the case study: the results obtained are discussed and the two methods are synthetically compared; finally, some conclusions are drawn in the last Section.

\section{Representation of aleatory and epistemic uncertainties in a two-level framework: fuzzy random variables}

In all generality, we consider an uncertain variable $Y$, whose uncertainty is described by the 96 Probability Distribution Function (PDF) $p^{Y}(y \mid \boldsymbol{\theta})$, where $\boldsymbol{\theta}=\left\{\theta_{1}, \theta_{2}, \ldots, \theta_{m}, \ldots, \theta_{P}\right\}$ is the vector of 7 the corresponding internal parameters. In a two-level framework, the parameters $\boldsymbol{\theta}$ are themselves 
affected by epistemic uncertainty [Limbourg and de Rocquigny, 2010]. In the present work, we describe these uncertainties by the (generally joint) possibility distribution $\pi^{\theta}(\boldsymbol{\theta})$ (it is straightforward to notice that in case the internal parameters $\left\{\theta_{1}, \theta_{2}, \ldots, \theta_{m}, \ldots, \theta_{P}\right\}$ are independent, then $\pi^{\theta}(\boldsymbol{\theta})$ is simply represented by a group of $P$ separate, marginal and independent (i.e., noninteractive) possibility distributions, i.e., $\left.\pi^{\theta}(\boldsymbol{\theta})=\left\{\pi^{\theta_{1}}\left(\theta_{1}\right), \pi^{\theta_{2}}\left(\theta_{2}\right), \ldots, \pi^{\theta_{m}}\left(\theta_{m}\right), \ldots, \pi^{\theta_{P}}\left(\theta_{P}\right)\right\}\right)$. A random variable $Y$ with possibilistic parameters $\boldsymbol{\theta}$ is a particular case of a Fuzzy Random Variable (FRV), i.e., of a random variable whose values are not real, but rather fuzzy numbers [Féron, 1976; Kwakernaak, 1978; Puri and Ralescu, 1986; Baudrit et al., 2008; Couso and Sanchez, 2008]. The corresponding Fuzzy Probability Distribution Function (FPDF) is here indicated as $\tilde{p}^{Y}(y \mid \boldsymbol{\theta})$.

For clarification by way of example, we may consider the generic uncertain variable $Y$ described by a Gumbel PDF, i.e., $Y \sim p^{Y}(y \mid \boldsymbol{\theta})=\operatorname{Gum}(\boldsymbol{\theta})=\operatorname{Gum}\left(\theta_{1}, \theta_{2}\right)=\operatorname{Gum}(\gamma, \delta)=p^{Y}(y \mid \gamma, \delta)$. Parameter $\delta=\theta_{2}$ (i.e., the scale paramter) is a fixed point-wise value ( $\delta=\theta_{2}=100$ ), whereas parameter $\gamma=\theta_{1}$ (i.e., the location parameter) is epistemically-uncertain. By hypothesis, the only information available on $\gamma=\theta_{1}$ is that it is defined on interval $\left[a_{\gamma}, b_{\gamma}\right]=[900,1300]$ and its most likely value is $c_{\gamma}=1100$. Notice that such information is not sufficient for assigning a single specific probability distribution to describe the epistemic uncertainty in parameter $\gamma=\theta_{1}$. In facts, such scarce information is actually compatible with a variety of probability distributions (e.g., truncated normal, lognormal, triangular, ...). To address this issue, this limited state of knowledge about $\gamma=\theta_{1}$ is here described by a triangular possibility distribution $\pi^{\gamma}(\gamma)$ with core $c_{\gamma}=1100$ and support $\left[a_{\gamma}, b_{\gamma}\right]=[900,1300]$ (Fig. 1, top left column) [Baudrit and Dubois, 2006]. Indeed, this representation is coherent with the information available, as it can be demonstrated that such possibility distribution "encodes" the family of all the probability distributions with mode $c_{\gamma}=1100$ and support $\left[a_{\gamma}, b_{\gamma}\right]=[900,1300]$ (obviously, this does not mean that the triangular possibility distribution is the only one with these characteristics, i.e., the only one able to encode such a 
probability family). In other words, one single possibility distribution generates in practice a 123 "bundle" of probability distributions with mode $c_{\gamma}=1100$ and support $\left[a_{\gamma}, b_{\gamma}\right]=[900,1300]$. The 124 reader is referred to [Baudrit and Dubois, 2006; Couso et al., 2001; Dubois et al., 2004] for further technical details and a formal proof of these statements.

126 In order to provide an additional practical interpretation of the possibility distribution $\pi^{\gamma}(\gamma)$ of $127 \gamma=\theta_{1}$, we can define its $\alpha$-cut sets $A_{\alpha}^{\gamma}=\left\{\gamma: \pi^{\gamma}(\gamma) \geq \alpha\right\}$, with $0 \leq \alpha \leq 1$. For example, $A_{0.5}^{\gamma}=$ $128[1000,1200]$ is the set of $\gamma$ values for which the possibility function is greater than or equal to 0.5 129 (dashed segment in Fig. 1, top left column). Notice that the $\alpha$-cut set $A_{\alpha}^{\gamma}$ of parameter $\gamma$ can be 130 interpreted also as the $(1-\alpha) \cdot 100 \%$ Confidence Interval (CI) for $\gamma$, i.e., the interval such that $131 P\left[\gamma \in A_{\alpha}^{\gamma}\right] \geq 1-\alpha$. For example, $A_{0}^{\gamma}=[900,1300]$ is the $(1-0) \cdot 100 \%=100 \%$ CI for $\gamma$, i.e., the 132 interval that contains the "true" value of $\gamma$ with certainty (solid segment in Fig. 1, top left column); $133 A_{0.5}^{\gamma}=[1000,1200]\left(\subset A_{0}^{\gamma}\right)$ is the $(1-0.5) \cdot 100 \%=50 \%$ CI (dashed segment in Fig. 1, top left 134 column $) ; A_{0.8}^{\gamma}=[1050,1150]\left(\subset A_{0.5}^{\gamma} \subset A_{0}^{\gamma}\right)$ is the $(1-0.8) \cdot 100 \%=20 \% \mathrm{CI}$, and so on. In this 135 view, the possibility distribution $\pi^{\gamma}(\gamma)$ can be interpreted as a set of nested CIs for parameter $\gamma$ 136 [Baudrit and Dubois, 2006; Couso et al., 2001; Dubois et al., 2004].

137 For each possibility (resp., confidence) level $\alpha$ (resp., $1-\alpha$ ) in [0, 1], a family of PDFs for $Y$, 138 namely $\left\{p^{Y}(y \mid \gamma, \delta)\right\}_{\alpha}$, can be generated by letting the epistemically-uncertain parameter $\gamma$ range 139 within the corresponding $\alpha$-cut set $A_{\alpha}^{\gamma}$, i.e., $\left\{p^{Y}(y \mid \gamma, \delta)\right\}_{\alpha}=\left\{p^{Y}(y \mid \gamma, \delta): \gamma \in A_{\alpha}^{\gamma}, \delta=100\right\}$. By way 140 of example, Fig. 1, top right column, shows four PDFs belonging to the family $\left\{p^{Y}(y \mid \gamma, \delta)\right\}_{\alpha=0}$ 141 (solid lines) and four PDFs belonging to the family $\left\{p^{Y}(y \mid \gamma, \delta)\right\}_{\alpha=0.5}$ (dashed lines).

142 In the same way, a bundle of Cumulative Distribution Functions (CDFs) for $Y$, namely $143\left\{F^{Y}(y \mid \gamma, \delta)\right\}_{\alpha}$, can be constructed by letting $\gamma$ range within $A_{\alpha}^{\gamma}$, i.e., $\left\{F^{Y}(y \mid \gamma, \delta)\right\}_{\alpha}=$ $144\left\{F^{Y}(y \mid \gamma, \delta): \gamma \in A_{\alpha}^{\gamma}, \sigma=100\right\}$. This family of CDFs (of level $\alpha$ ) is bounded above and below by the 
upper and lower CDFs, $\bar{F}_{\alpha}^{Y}(y)$ and $\underline{F}_{\alpha}^{Y}(y)$, defined as $\bar{F}_{\alpha}^{Y}(y)=\sup _{\gamma \in A_{\alpha}^{\gamma}}\left\{F^{Y}(y \mid \gamma, \delta=100)\right\}$ and $\underline{F}_{\alpha}^{Y}(y)=\inf _{\gamma \in A_{\alpha}^{\gamma}}\left\{F^{Y}(y \mid \gamma, \delta=100)\right\}$, respectively. Since $\pi^{\gamma}(\gamma)$ can be interpreted as a set of nested CIs for parameter $\gamma$ (see above), it can be argued that the $\alpha$-cuts of $\pi^{\gamma}(\gamma)$ induce also a set of nested 148 pairs of $\operatorname{CDFs}\left\{\left(\underline{F}_{\alpha}^{Y}(y), \bar{F}_{\alpha}^{Y}(y)\right): 0 \leq \alpha \leq 1\right\}$ which bound the "true" $\operatorname{CDF} F^{Y}(y)$ of $Y$ with confidence 149 larger than or equal to $(1-\alpha)$, i.e., $P\left[\underline{F}_{\alpha}^{Y}(y) \leq F^{Y}(y) \leq \bar{F}_{\alpha}^{Y}(y)\right] \geq 1-\alpha$, with $0 \leq \alpha \leq 1$ [Baudrit et al., 2007 and 2008]. In passing, notice that the upper and lower CDFs (of level $\alpha$ ), $\bar{F}_{\alpha}^{Y}(y)$ and $\underline{F}_{\alpha}^{Y}(y)$, can be referred to as the plausibility and belief functions (of level $\alpha$ ) of the set $Z=(-\infty, y]$, i.e., $\bar{F}_{\alpha}^{Y}(y)=P l_{\alpha}^{Y}(Z)$ and $\underline{F}_{\alpha}^{Y}(y)=B e l_{\alpha}^{Y}(Z)$, respectively. For illustration purposes, Fig. 1, bottom row, shows the bounding upper and lower CDFs of $Y, \bar{F}_{\alpha}^{Y}(y)=P l_{\alpha}^{Y}(Z)$ and $\underline{F}_{\alpha}^{Y}(y)=\operatorname{Bel}_{\alpha}^{Y}(Z)$, built in correspondence of the $\alpha$-cuts of level $\alpha=0$ (solid lines), 0.5 (dashed lines) and 1 (dot-dashed line) of the possibility distribution $\pi^{\gamma}(\gamma)$ of parameter $\gamma$ (Fig. 1, top left column).

Finally, the set of nested pairs of CDFs $\left\{\left(\underline{F}_{\alpha}^{Y}(y), \bar{F}_{\alpha}^{Y}(y)\right): 0 \leq \alpha \leq 1\right\}=\left\{\left(\operatorname{Bel}_{\alpha}^{Y}(Z), P l_{\alpha}^{Y}(Z)\right): 0 \leq \alpha \leq 1\right\}$, $Z=(-\infty, y]$, can be synthesized into a single pair of plausibility and belief functions as $P l^{Y}(Z)=\int_{0}^{1} P l_{\alpha}^{Y}(Z) d \alpha$ and $\operatorname{Bel}^{Y}(Z)=\int_{0}^{1} \operatorname{Bel}_{\alpha}^{Y}(Z) d \alpha$, respectively (dotted lines in Fig. 1, bottom row): in other words, $P l^{Y}(Z)$ and $\operatorname{Bel}^{Y}(Z)$ are obtained by averaging the different nested plausibility and belief functions (i.e., $\left.\left\{\left(\operatorname{Bel}_{\alpha}^{Y}(Z), P l_{\alpha}^{Y}(Z)\right): 0 \leq \alpha \leq 1\right\}\right)$ generated at different possibility levels $\alpha \in[0,1]$ (i.e., by averaging the different contributions to the plausibility and belief functions produced by different $\alpha$-cuts of the epistemic parameter $\gamma$ ). The plausibility and belief functions $\operatorname{Pl}^{Y}(Z)$ and $\operatorname{Bel}^{Y}(Z), Z=(-\infty, y]$, are shown to represent the "best bounds" for the "true" CDF $F^{Y}(y)$ of the uncertain variable $Y$ [Ralescu, 2002; Baudrit et al., 2007 and 2008; Couso 
166 Further details about FRVs are not given here for the sake of brevity: the interested reader is referred to the cited references.

Bayesian update of the possibilistic parameters of aleatory probability distributions

In this Section, we present the methods employed in this study for updating, in a Bayesian probability distributions, as new information/evidence (e.g., data) becomes available. In this view, let $\pi^{\theta}(\boldsymbol{\theta})$ be the (joint) prior possibility distribution for the parameters $\boldsymbol{\theta}=\left[\theta_{1}, \theta_{2}, \ldots, \theta_{m}, \ldots, \theta_{P}\right]$ of the PDF $p^{Y}(y \mid \boldsymbol{\theta})$ of variable $Y$ (built on the basis of a priori subjective engineering knowledge and/or data). For example, in the risk assessment context of this paper $Y$ may represent the yearly maximal water flow of a river described by a Gumbel distribution: thus, $Y \sim p^{Y}(y \mid \boldsymbol{\theta})=G u m(\boldsymbol{\theta})=$

$\operatorname{Gum}\left(\theta_{1}, \theta_{2}\right)=\operatorname{Gum}(\gamma, \delta)=p^{Y}(y \mid \gamma, \delta)$ and $\pi^{\theta}(\boldsymbol{\theta})=\pi^{\gamma, \delta}(\gamma, \delta)$. Moreover, let $\boldsymbol{y}=\left[y_{1}, y_{2}, \ldots, y_{k}, \ldots, y_{D}\right]$ be a vector of $D$ observed pieces of data representing the new information/evidence available for the analysis: referring to the example above, $\boldsymbol{y}$ may represent a vector of $D$ values collected over a long period time (e.g., many years) of the yearly maximal water flow of the river under analysis. The objective of the Bayesian analysis is to update the a priori representation $\pi^{\theta}(\boldsymbol{\theta})=\pi^{\gamma, \delta}(\gamma, \delta)$ of $\boldsymbol{\theta}=[\gamma, \delta]$ on the basis of the new evidence acquired, i.e., to

calculate the posterior possibility distribution $\pi^{\theta}(\boldsymbol{\theta} \mid \boldsymbol{y})$ (i.e., $\pi^{\gamma, \delta}(\gamma, \delta \mid \boldsymbol{y})$ ) of $\boldsymbol{\theta}$ after $\boldsymbol{y}$ is obtained.

In the present paper, two methods are considered to this aim: the purely possibilistic method and the hybrid probabilistic and possibilistic approach. 


\section{Purely possibilistic approach}

The purely possibilistic method (hereafter also referred to as 'Approach A' for brevity) is based on a purely possibilistic counterpart of the classical, probabilistic Bayes' theorem [Dubois and Prade, 1997; Lapointe and Bobée, 2000]:

$\pi^{\theta}(\boldsymbol{\theta} \mid \boldsymbol{y})=\frac{\pi_{L}^{\theta}(\boldsymbol{\theta} \mid \boldsymbol{y}) \cdot \pi^{\theta}(\boldsymbol{\theta})}{\sup _{\boldsymbol{\theta}}\left\{\pi_{L}^{\theta}(\boldsymbol{\theta} \mid \boldsymbol{y}) \cdot \pi^{\theta}(\boldsymbol{\theta})\right\}}$

where $\pi_{L}^{\theta}(\boldsymbol{\theta} \mid \boldsymbol{y})$ is the possibilistic likelihood of the parameter vector $\boldsymbol{\theta}$ given the newly observed data $\boldsymbol{y}$, and quantities $\pi^{\theta}(\boldsymbol{\theta} \mid \boldsymbol{y})$ and $\pi^{\theta}(\boldsymbol{\theta})$ are defined above. Notice that $\sup _{\boldsymbol{\theta}}\left\{\pi_{L}^{\theta}(\boldsymbol{\theta} \mid \boldsymbol{y}) \cdot \pi^{\boldsymbol{\theta}}(\boldsymbol{\theta})\right\}$ is a normalization factor such that $\sup _{\boldsymbol{\theta}}\left\{\pi^{\theta}(\boldsymbol{\theta} \mid \boldsymbol{y})\right\}=1$, as required by possibility theory [Dubois, 2006].

It is worth mentioning that forms of the possibilistic Bayes' theorem alternative to (1) can be constructed as a result of other definitions of the operation of 'conditioning' with possibility distributions: the reader is referred to [Dubois and Prade, 1997; Lapointe and Bobée, 2000] for technical details. In this paper, expression (1) has been chosen because "it satisfies desirable properties of the revision process and lead to continuous posterior distributions" [Lapointe and Bobée, 2000].

The possibilistic likelihood $\pi_{L}^{\theta}(\boldsymbol{\theta} \mid \boldsymbol{y})$ is here obtained by transforming the classical probabilistic likelihood function $L^{\theta}(\boldsymbol{\theta} \mid \boldsymbol{y})$ through normalization, i.e., $\pi_{L}^{\theta}(\boldsymbol{\theta} \mid \boldsymbol{y})=\frac{L^{\theta}(\boldsymbol{\theta} \mid \boldsymbol{y})}{\sup _{\boldsymbol{\theta}}\left\{L^{\theta}(\boldsymbol{\theta} \mid \boldsymbol{y})\right\}}$ [Anoop et al., 2006] (obviously, $L^{\boldsymbol{\theta}}(\boldsymbol{\theta} \mid \boldsymbol{y})=\prod_{k=1}^{D} p^{Y}\left(y_{k} \mid \boldsymbol{\theta}\right)$ in the case the observations $\left\{y_{k}: k=1,2, \ldots, D\right\}$ are independent and identically distributed). This choice has been made for the following main reasons:

i. the transformation is simple and can be straightforwardly applied to any distribution [Anoop et al., 2006];

ii. the resulting possibilistic likelihood is very closely related to the classical, purely probabilistic one (which is theoretically well-grounded) by means of the simple and direct 
operation of normalization that preserves the "original structure" of the experimental evidence;

213 iii. it can be easily verified that the resulting possibilistic likelihood keeps the sequential nature of the updating procedure typical of the standard Bayes' theorem;

iv. the operation of likelihood normalization finds also theoretical justifications in some recent works of literature (see the brief discussion below) [Denoeux, 2014; Moral, 2014].

217 However, two considerations are in order with respect to this choice. First, it has to be admitted that 218 the resulting possibility distributions do not in general adhere to the probability-possibility consistency principle [Dubois and Prade, 1980]. Second, it has to be remembered that the probabilistic likelihood function $L^{\theta}(\boldsymbol{\theta} \mid \boldsymbol{y})$ is not a probability distribution: in this view, from a rigorous mathematical viewpoint, speaking of probability/possibility transformation for it would be wrong. On the other side, from the practical engineering viewpoint of interest to the present paper, an operation of normalization can be performed (i.e., $L^{\theta}(\boldsymbol{\theta} \mid \boldsymbol{y}) / \int_{\boldsymbol{\theta}} L^{\boldsymbol{\theta}}(\boldsymbol{\theta} \mid \boldsymbol{y}) d \boldsymbol{\theta}$ ) in order to "technically" provide it with the "properties" of a probability distribution function.

It is worth noting that other techniques of transformation of probability density functions into possibility distributions exist, but the corresponding details are not given here for brevity sake: the interested reader is referred to [Dubois et al., 1993, 2004 and 2008; Flage et al., 2010 and 2013] for some proposed techniques, e.g., the principle of maximum specificity [Dubois et al., 1993] and the principle of minimal commitment [Dubois et al., 2008]. Also, it has to be noticed that techniques are also available to construct possibility distributions (and, thus, possibilistic likelihood functions) 231 directly from rough experimental data (i.e., without resorting to probability-possibility 232 transformations): see [Masson and Denoeux, 2006; Mauris, 2008; Hou and Yang, 2010; Serrurier 233 and Prade, 2011] for more details. Finally, for a thorough theoretical justification of a "possibilistic 234 vision" of the likelihood the reader is referred to: e.g., [Dubois et al., 1997], where possibility measures are considered as the supremum of a family of likelihood functions; [Denoeux, 2014], 
where the evidence about a parameter (after observing a piece of data) is represented by a

consonant "likelihood based" belief function, whose contour function equals the normalized likelihood function (see above): in the paper, this is also rigorously derived from three basic principles, i.e., the likelihood principle [Edwards, 1992], compatibility with Bayes' rule and the minimal commitment principle [Smets, 1993]; and finally [Moral, 2014], where the approach by [Denoeux, 2014] is discussed and the issue of representing likelihood information is taken from the point of view of imprecise probabilities.

\section{Hybrid probabilistic and possibilistic approach}

The hybrid probabilistic and possibilistic method (hereafter also called 'Approach B' for brevity) is based on the construction of a Fuzzy Probability Distribution Function (FPDF) to be used as a 'fictitious' prior for the epistemically-uncertain parameters $\boldsymbol{\theta}$ of the PDF $p^{Y}(y \mid \boldsymbol{\theta})$ of the uncertain variable $Y$ : in other words, a fictitious (artificial) probabilistic function has to be 'superimposed' onto the purely possibilistic prior $\pi^{\theta}(\boldsymbol{\theta})$ that has to be updated. In more detail, let $\tilde{p}^{\theta}(\boldsymbol{\theta} \mid \boldsymbol{\varphi})$ be the

fictitious (prior) FPDF of $\boldsymbol{\theta}$, constructed by the superimposition of an (arbitrarily selected)

252 are independent, then $p^{\theta}(\boldsymbol{\theta} \mid \boldsymbol{\varphi})=\prod_{m=1}^{P} p^{\theta_{m}}\left(\theta_{m} \mid \boldsymbol{\varphi}_{m}\right)$, with $\boldsymbol{\varphi}_{m}=\left[\boldsymbol{\phi}_{m 1}, \phi_{m 2}, \ldots, \phi_{m N_{m}}\right], m=1,2, \ldots$ $P$; also, $\pi^{\varphi}(\varphi)$ is expressed as $\pi^{\varphi}(\varphi)=\left\{\pi^{\varphi_{1}}\left(\varphi_{1}\right), \pi^{\varphi_{2}}\left(\varphi_{2}\right), \ldots, \pi^{\varphi_{m}}\left(\varphi_{m}\right), \ldots, \pi^{\varphi_{P}}\left(\varphi_{P}\right)\right\}$. In addition, if also the possibilistic parameters $\phi_{m 1}, \phi_{m 2}, \ldots, \phi_{m n_{m}}$ of the fictitious $\operatorname{PDF} p^{\theta_{m}}\left(\theta_{m} \mid \boldsymbol{\varphi}_{m}\right), m=1,2$, $\ldots, P$, are independent, then $\left.\pi^{\varphi_{m}}\left(\boldsymbol{\varphi}_{m}\right)=\left\{\pi^{\varphi_{m 1}}\left(\phi_{m 1}\right), \pi^{\varphi_{m 2}}\left(\phi_{m 2}\right), \ldots, \pi^{\varphi_{m N_{m}}}\left(\phi_{m N_{m}}\right)\right\}\right)$. As before, let $Y \sim$ $p^{Y}(y \mid \boldsymbol{\theta})=\operatorname{Gum}(\gamma, \delta)=p^{Y}(y \mid \gamma, \delta)$, with $\boldsymbol{\theta}=\left[\theta_{1}, \theta_{2}\right]=[\gamma, \delta]$ epistemically-uncertain. For simplicity, we consider $\theta_{1}=\gamma$ and $\theta_{2}=\delta$ independent, such that $\pi^{\theta}(\boldsymbol{\theta})=\left\{\pi^{\gamma}(\gamma), \pi^{\delta}(\delta)\right\}$ and that 
258 the (fictitious) PDF $p^{\boldsymbol{\theta}}(\boldsymbol{\theta} \mid \boldsymbol{\varphi})$ can be written as $\prod_{m=1}^{P} p^{\theta_{m}}\left(\theta_{m} \mid \boldsymbol{\varphi}_{m}\right)=p^{\theta_{1}}\left(\theta_{1} \mid \boldsymbol{\varphi}_{1}\right) \cdot p^{\theta_{2}}\left(\theta_{2} \mid \boldsymbol{\varphi}_{2}\right)=$ $259 p^{\gamma}\left(\gamma \mid \varphi_{\gamma}\right) \cdot p^{\delta}\left(\delta \mid \varphi_{\delta}\right)$. By hypothesis, the analyst arbitrarily selects Normal distributions to 260 represent $p^{\gamma}\left(\gamma \mid \boldsymbol{\varphi}_{\gamma}\right)$ and $p^{\delta}\left(\delta \mid \boldsymbol{\varphi}_{\delta}\right)$, i.e., $p^{\gamma}\left(\gamma \mid \boldsymbol{\varphi}_{\gamma}\right)=N\left(\boldsymbol{\varphi}_{\gamma}\right)=N\left(\mu_{\gamma}, \sigma_{\gamma}\right)$ and $p^{\delta}\left(\delta \mid \boldsymbol{\varphi}_{\delta}\right)=$ $261 N\left(\varphi_{\delta}\right)=N\left(\mu_{\delta}, \sigma_{\delta}\right)$, respectively (Normal and Uniform distributions are indicated as good choices 262 for $p^{\theta}(\boldsymbol{\theta} \mid \boldsymbol{\varphi})$ by [Beer, 2009a; Stein and Beer, 2011; Stein et al., 2013]). Then, parameters $263 \boldsymbol{\varphi}_{\gamma}=\left[\mu_{\gamma}, \sigma_{\gamma}\right]$ and $\boldsymbol{\varphi}_{\delta}=\left[\mu_{\delta}, \sigma_{\delta}\right]$ are represented by the possibility distributions $264 \pi^{\varphi_{\gamma}}=\left\{\pi^{\mu_{\gamma}}\left(\mu_{\gamma}\right), \pi^{\sigma_{\gamma}}\left(\sigma_{\gamma}\right)\right\}$ and $\pi^{\varphi_{\delta}}=\left\{\pi^{\mu_{\delta}}\left(\mu_{\delta}\right), \pi^{\sigma_{\delta}}\left(\sigma_{\delta}\right)\right\}$ (by so doing, the fictitious prior FPDF $265 \tilde{p}^{\theta}(\boldsymbol{\theta} \mid \boldsymbol{\varphi})=\tilde{p}^{\theta_{1}}\left(\theta_{1} \mid \boldsymbol{\varphi}_{1}\right) \cdot \tilde{p}^{\theta_{2}}\left(\theta_{2} \mid \boldsymbol{\varphi}_{2}\right)=\tilde{p}^{\gamma}\left(\gamma \mid \boldsymbol{\varphi}_{\gamma}\right) \cdot \tilde{p}^{\delta}\left(\delta \mid \boldsymbol{\varphi}_{\delta}\right)$ is constructed). These possibility 266 distributions should be properly selected by the analyst so as to reflect as closely as possible the 267 structure of the 'real' prior possibility distribution $\pi^{\theta}(\boldsymbol{\theta})=\left\{\pi^{\gamma}(\gamma), \pi^{\delta}(\delta)\right\}$ that has to be updated: 268 for example, $\pi^{\varphi_{\gamma}}$ and $\pi^{\varphi_{\delta}}$ could be identified by 'imposing' that the expected value of the FPDF $269 \tilde{p}^{\theta}(\boldsymbol{\theta} \mid \varphi)$ corresponds to the real prior possibility distribution $\pi^{\theta}(\boldsymbol{\theta})$, i.e., $E_{\varphi}\left[\tilde{p}^{\theta}(\boldsymbol{\theta} \mid \boldsymbol{\varphi})\right]=\pi^{\theta}(\boldsymbol{\theta})$ 270 or, in this case, $E_{\varphi_{\gamma}}\left[\tilde{p}^{\gamma}\left(\gamma \mid \varphi_{\gamma}\right)\right]=\pi^{\gamma}(\gamma)$ and $E_{\varphi_{\delta}}\left[\tilde{p}^{\delta}\left(\delta \mid \varphi_{\delta}\right)\right]=\pi^{\delta}(\delta)$.

271 In extreme synthesis, the method relies on the hybrid combination of (i) Fuzzy Interval Analysis 272 (FIA) to process the uncertainty described by possibility distributions and (ii) repeated Bayesian 273 updating of the uncertainty represented by probability distributions [Beer, 2009a; Stein and Beer, 274 2011; Stein et al., 2013]. In more details, the algorithm proceeds as follows:

$275 \quad$ 1. set $\alpha=0$;

2. select the $\alpha$-cut $A_{\alpha}^{\varphi}$ of the possibility distribution $\pi^{\varphi}(\varphi)$ of vector $\varphi$ of the parameters of the (fictitious) prior $\operatorname{PDF} p^{\theta}(\boldsymbol{\theta} \mid \boldsymbol{\varphi})$;

3. letting the parameter vector $\varphi$ range within the corresponding $\alpha$-cut $A_{\alpha}^{\varphi}$ identified at step 2 . above, generate a family of (fictitious) prior PDFs $\left\{p^{\theta}(\boldsymbol{\theta} \mid \boldsymbol{\varphi})\right\}_{\alpha}=\left\{p^{\theta}(\boldsymbol{\theta} \mid \boldsymbol{\varphi}): \boldsymbol{\varphi} \in A_{\alpha}^{\varphi}\right\}$. This is 
empirically done by (i) randomly or deterministically selecting a finite number $T$ (e.g., $T=$ 100 in this paper) of parameter vectors $\varphi_{l, \alpha}, l=1,2, \ldots, T$, in $A_{\alpha}^{\varphi}$ and (ii) evaluating $p^{\theta}(\boldsymbol{\theta} \mid \boldsymbol{\varphi})$ in correspondence of these vectors, i.e., $\left\{p^{\theta}(\boldsymbol{\theta} \mid \boldsymbol{\varphi})\right\}_{\alpha} \approx\left\{p^{\theta}\left(\boldsymbol{\theta} \mid \boldsymbol{\varphi}_{l, \alpha}\right): l=1,2, \ldots, T\right\}$

4. apply the classical probabilistic Bayes theorem to each (fictitious) prior $\operatorname{PDF} p^{\theta}\left(\boldsymbol{\theta} \mid \boldsymbol{\varphi}_{l, \alpha}\right)$ generated at step 3. above to get the corresponding posterior PDF $p_{l, \alpha}^{\theta}(\boldsymbol{\theta} \mid \boldsymbol{y})$ :

$$
p_{l, \alpha}^{\boldsymbol{\theta}}(\boldsymbol{\theta} \mid \boldsymbol{y})=\frac{L^{\boldsymbol{\theta}}(\boldsymbol{\theta} \mid \boldsymbol{y}) \cdot p\left(\boldsymbol{\theta} \mid \boldsymbol{\varphi}_{l, \alpha}\right)}{\int_{\boldsymbol{\theta}} L^{\boldsymbol{\theta}}(\boldsymbol{\theta} \mid \boldsymbol{y}) \cdot p\left(\boldsymbol{\theta} \mid \boldsymbol{\varphi}_{l, \alpha}\right) d \boldsymbol{\theta}}, l=1,2, \ldots, T
$$

This is equivalent to generating a family $\left\{p^{\theta}(\boldsymbol{\theta} \mid \boldsymbol{y})\right\}_{\alpha}$ of posterior PDFs for $\boldsymbol{\theta}$, i.e., $\left\{p^{\theta}(\boldsymbol{\theta} \mid \boldsymbol{y})\right\}_{\alpha} \approx\left\{p_{l, \alpha}^{\theta}(\boldsymbol{\theta} \mid \boldsymbol{y}): l=1,2, \ldots, T\right\}$

5. calculate the expected value of each posterior $\operatorname{PDF} p_{l, \alpha}^{\theta}(\boldsymbol{\theta} \mid \boldsymbol{y})$ generated at step 4. above to obtain a point estimate $\boldsymbol{\theta}_{l, \alpha} \mid \boldsymbol{y}$ for the epistemically-uncertain parameter vector $\boldsymbol{\theta}$ [Stein et al., 2013]:

$$
\boldsymbol{\theta}_{l, \alpha} \mid \boldsymbol{y}=E_{\theta}\left[p_{l, \alpha}^{\theta}(\boldsymbol{\theta} \mid \boldsymbol{y})\right], l=1,2, \ldots, T
$$

6. take the hull enveloping the $T$ point estimates $\boldsymbol{\theta}_{l, \alpha} \mid \boldsymbol{y}, l=1,2, \ldots, T$, as the ( $P$-dimensional) $\alpha$-cut $A_{\alpha}^{\theta \mid y}$ of the (joint $P$-dimensional) posterior possibility distribution $\pi^{\theta}(\boldsymbol{\theta} \mid \boldsymbol{y})$ of $\boldsymbol{\theta}$. By way of example and for illustration purposes, Fig. 2 shows the identification of a (twodimensional) $\alpha$-cut $A_{\alpha}^{\theta \mid y}=A_{\alpha}^{\theta_{1}, \theta_{2} \mid y}$ (solid line) as the contour enclosing $T=20$ point estimates $\boldsymbol{\theta}_{l, \alpha} \mid \boldsymbol{y}, l=1,2, \ldots, 20$ (dots), in the simplified case of $P=2$ parameters $\theta_{1}$ and $\theta_{2}$

7. if $\alpha<1$, then set $\alpha=\alpha+\Delta \alpha$ (e.g., $\Delta \alpha=0.05$ in this paper) and return to step 2. above; otherwise, stop the algorithm. 
The (joint $P$-dimensional) posterior possibility distribution $\pi^{\theta}(\boldsymbol{\theta} \mid \boldsymbol{y})$ for the epistemically-uncertain

300 parameter vector $\boldsymbol{\theta}$ is empirically constructed as the (discrete) collection of the $\alpha$-cuts $A_{\alpha}^{\theta \mid y}, \alpha=0$, $0.05, \ldots, 0.95,1$, found at step 6 . above.

It is worth noting that the application of both approaches $\mathrm{A}$ and $\mathrm{B}$ always produces a joint $P$ dimensional posterior possibility distribution $\pi^{\theta}(\boldsymbol{\theta} \mid \boldsymbol{y})$ (whatever the state of dependence between the priors), characterized by $P$-dimensional $\alpha$-cuts $A_{\alpha}^{\theta \mid y}$, with $0<\alpha<1$ : as a consequence, there is an interactive dependence between the values that parameters $\left\{\theta_{m}: m=1,2, \ldots, P\right\}$ can take when ranging within a given $\alpha$-cut $A_{\alpha}^{\theta \mid y}$ : for example, in Fig. 2 it is impossible that parameter $\theta_{1}$ takes on low values and parameter $\theta_{2}$ takes on high values at the same time.

From $\pi^{\theta}(\boldsymbol{\theta} \mid \boldsymbol{y})$ it is straightforward to obtain the marginal posterior possibility distribution $\pi^{\theta_{m}}\left(\theta_{m} \mid \boldsymbol{y}\right)$ for each parameter $\theta_{m}$ as $\pi^{\theta_{m}}\left(\theta_{m} \mid \boldsymbol{y}\right)=\max _{\theta_{j} \in \mathfrak{\Re}, j \neq m}\left\{\pi^{\boldsymbol{\theta}}(\boldsymbol{\theta} \mid \boldsymbol{y})\right\}, \forall \theta_{m} \in \mathfrak{R}, m=1,2, \ldots, P$ [Baudrit et al., 2006]: $\pi^{\theta_{m}}\left(\theta_{m} \mid \boldsymbol{y}\right)$ is the projection of $\pi^{\theta}(\boldsymbol{\theta} \mid \boldsymbol{y})$ onto the $m$-th axis. The (onedimensional) $\alpha$-cut $A_{\alpha}^{\theta_{m} \mid \boldsymbol{y}}=\left[\underline{\theta}_{m, \alpha}\left|y, \bar{\theta}_{m, \alpha}\right| y\right]$ of the marginal possibility distribution $\pi^{\theta_{m}}\left(\theta_{m} \mid \boldsymbol{y}\right)$ is then related to the (P-dimensional) $\alpha$-cut $A_{\alpha}^{\theta \mid y}$ of the joint possibility distribution $\pi^{\theta}(\boldsymbol{\theta} \mid \boldsymbol{y})$ by the following straightforward relation, i.e., $A_{\alpha}^{\theta_{m} \mid y}=\left[\underline{\theta}_{m, \alpha}\left|y, \bar{\theta}_{m, \alpha}\right| y\right]=\left[\min _{\boldsymbol{\theta} \in A_{\alpha}^{\theta \mid y}}\left\{\theta_{m}\right\}, \max _{\theta \in A_{\alpha}^{\theta \mid y}}\left\{\theta_{m}\right\}\right]$. In this view, notice that the use of the $P$-dimensional $\alpha$-cut $A_{\alpha, \text { Cart }}^{\theta \mid y}$ constructed by the Cartesian product of the (one-dimensional) $\alpha$-cuts $A_{\alpha}^{\theta_{m} \mid y}$ of the marginal distributions, $m=1,2, \ldots, P$ (i.e., $A_{\alpha, \text { Cart }}^{\theta \mid y}=A_{\alpha}^{\theta_{1} \mid y}$ $\mathrm{x} A_{\alpha}^{\theta_{2} \mid y} \times \ldots \times A_{\alpha}^{\theta_{m} \mid y} \mathrm{x} \ldots \mathrm{x} A_{\alpha}^{\theta_{\rho} \mid y}$ ) would (incorrectly) imply independence between the posterior estimates of the parameters $\left\{\theta_{m}: m=1,2, \ldots, P\right\} ;$ however, since $A_{\alpha, \text { Cart }}^{\theta \mid y}$ completely contains $A_{\alpha}^{\theta \mid y}$ (i.e., by definition $A_{\alpha}^{\theta \mid y} \subset A_{\alpha, \text { Cart }}^{\theta \mid y}$ ), then conservatism would be still guaranteed [Stein et al., 2013]. For illustration purposes and with reference to the example above, Fig. 2 shows also the two- 
dimensional $\alpha$-cut $A_{\alpha, \text { Cart }}^{\theta_{1}, \theta_{2} \mid y}$ (dashed line) generated by the Cartesian product of the (one-dimensional) $\alpha$-cuts $A_{\alpha}^{\theta_{1} \mid y}$ and $A_{\alpha}^{\theta_{2} \mid y}$, i.e., $A_{\alpha, \text { Cart }}^{\theta_{1}, \theta_{2} \mid y}=A_{\alpha}^{\theta_{1} \mid y} \mathrm{x} A_{\alpha}^{\theta_{2} \mid y}$.

\section{Fig. 2}

The characteristics of the two approaches are summarized in Table 1. Notice that both methods relies on arbitrary assumptions about either the prior or the likelihood functions: in the purely possibilistic approach (A) the "original" possibilistic prior is employed, but a possibilistic likelihood function has to be constructed (e.g., by probability-possibility transformations, directly from rough experimental data and/or by resorting to the guidelines provided by [Dubois et al., 1997; Denoeux, 2014]); instead, in the hybrid method (B) the original probabilistic likelihood function is used, but a "fictitious" prior Fuzzy Probability Distribution Function needs to be identified by superimposing an arbitrarily selected probabilistic PDF onto the "original" possibilistic prior that has to be updated.

\section{Table 1.}

A final consideration is in order with respect to the two approaches here outlined. In the hybrid probabilistic-possibilistic framework of interest to the present paper, the knowledge a priori available on the parameters $\boldsymbol{\theta}=\left[\theta_{1}, \theta_{2}, \ldots, \theta_{m}, \ldots, \theta_{P}\right]$ of a given (aleatory) probability model $p^{Y}(y \mid \boldsymbol{\theta})$ is described by the prior possibility distribution function $\pi^{\theta}(\boldsymbol{\theta})$. As detailed in the previous Section, the possibilistic approach is particularly suitable to address those situations where the information a priori available on $\boldsymbol{\theta}$ is scarce and imprecise, i.e., not sufficient for assigning a single specific probability distribution to (describe the epistemic uncertainty in) $\boldsymbol{\theta}$. Actually, the possibilistic function $\pi^{\theta}(\boldsymbol{\theta})$ is in practice "equivalent" to the family of all those probability distributions (of possibly different shapes) that are coherent with the scarce information available on $\boldsymbol{\theta}$. On the other hand, it is worth mentioning that in a classical purely probabilistic framework, imprecision in prior information about $\boldsymbol{\theta}$ can be also accounted for by means of the so-called 
Hierarchical Bayes approach. Hierarchical Bayes is so-named because it utilizes hierarchical or

349 multistage prior distributions [Gelman, 2006; Gelman et al., 2008; Congdon, 2010; Kelly and 350 Smith, 2011; Chung et al., 2015; Shirley and Gelman, 2015]. To develop a hierarchical model for $351 \boldsymbol{\theta}$, we need to specify a first-stage prior (say, $p^{\theta}(\boldsymbol{\theta} \mid \boldsymbol{\varphi})$ ), which is often of a particular functional form, often a conjugate prior. However, analysts find it difficult to express their incertitude numerically at all, much less as particular probability distributions. Thus, a higher-dimensional model is defined to represent such (epistemic) uncertainty: in particular, we need to specify an "additional" prior distribution (say, $p^{\varphi}(\varphi \mid \omega)$ ) on the first-stage parameters $\varphi$. Distribution $p^{\varphi}(\varphi \mid \omega)$ is called the second-stage prior, or hyper-prior. This way of proceeding amounts to generating a 'parametric' family $\left\{p^{\theta}(\boldsymbol{\theta} \mid \boldsymbol{\varphi})\right\}$ of (first-stage prior) probability distributions for $\boldsymbol{\theta}$, all obtained in correspondence of different possible values of the first-stage parameters $\varphi$ (described by hyper-prior $\left.p^{\varphi}(\varphi \mid \omega)\right)$. This method has been investigated in the field of social and behavioral sciences with the main aim of treating hierarchical data with different levels of variables in the same statistical model. For example, the hierarchical data for sociological survey analysis include measurements from individuals with different historical, geographic, or economic variables. To this end, the hierarchical modeling was proposed to account for the different grouping or times at which data are measured [Gill, 2002]. In addition, a common application of hierarchical Bayes analysis in the Probabilistic Risk Assessment (PRA) of nuclear power plants has been as a model of variability among data sources, for example variability in Emergency Diesel Generator (EDG) performance across different plants, or across time [Siu and Kelly, 1998; Atwood et al., 2003; Kelly and Smith, 2009]. Finally, similar analogy can be made for the collected measurements from a structure under different ambient and environmental conditions. This framework has been recently implemented for uncertainty quantification applications in structural dynamics [Behmanesh et al., 2015; Ballesteros et al., 2014]. Although hierarchical Bayes can address the issue of imprecise prior information by means of multi-level models, the following conceptual and practical considerations should be made 
about its applicability: (i) in principle, one could define even higher dimensional models to represent the uncertainty. Some analysts have attempted three- and even four-level models [Jaworska and Aldenberg, 2000], but this hardly seems a workable solution when the complexity of the analysis is the primary problem; (ii) in addition, "if concern about the uncertainty in parameters can in principle drive the analysis to a higher level, one could fall into an insoluble infinite cascade" [Ferson, 2005]; (iii) finally, both first- and second-stage priors, $p^{\theta}(\boldsymbol{\theta} \mid \boldsymbol{\varphi})$ and $p^{\varphi}(\boldsymbol{\varphi} \mid \boldsymbol{\omega})$, respectively, are often of a particular functional form, which forces the analyst to make "overlyoptimistic" and excessively precise statements about parameter uncertainties, even when the information and data available are scarce and/or vague, i.e., not sufficient for assigning a single specific probability distribution. In such cases, non-probabilistic methods (e.g., intervals, possibility distributions or Dempster-Shafer structures from evidence theory) have been shown to provide reliable and robust results [Ferson et al., 2003; Beer et al., 2013a]. On the basis of considerations (i)-(iii) above and given that the objective of the paper is the comparison of methods for the update of possibilistic parameters (of aleatory probability distributions), Hierarchical Bayes approaches are not considered in the present work.

\section{Case study: flood protection risk-based design}

The case study deals with the design of a protection dike in a residential area closely located to a river with potential risk of floods. Two issues of concern are: (i) high construction and annual maintenance costs of the dike; (ii) uncertainty in the natural phenomenon of flooding. Then, the different design options must be evaluated within a flooding risk analysis framework accounting for uncertainty.

\section{The model}

The maximal water level of the river (i.e., the output variable of the model, $Z_{c}$ ) is given as a function of several (and some uncertain) parameters (i.e., the inputs to the model) [Limbourg and de Rocquigny, 2010]: 
399 where: $Y_{1}=Q$ is the yearly maximal water discharge $\left[\mathrm{m}^{3} / \mathrm{s}\right] ; Y_{2}=Z_{m}$ and $Y_{3}=Z_{v}$ are the riverbed 400 levels [m asl] at the upstream and downstream parts of the river under investigation, respectively; $Y_{4}$ $401=K_{s}$ is the Strickler friction coefficient; $Y_{5}=B$ and $Y_{6}=L$ are the width and length of the river 402 part [m], respectively. Quantities $Y_{5}=B(=300 \mathrm{~m})$ and $Y_{6}=L(=5000 \mathrm{~m})$ are constant parameters, 403 whereas quantities $Y_{1}=Q, Y_{2}=Z_{m}, Y_{3}=Z_{v}, Y_{4}=K_{s}$ are uncertain variables.

404 405 406 407 408 409

\section{The input variables: physical description and representation of the associated uncertainty}

The $n=4$ input variables $Y_{i}, i=1,2,3,4$, are affected by aleatory and epistemic uncertainties. The aleatory part of the uncertainty is described by probability distributions of defined shape. The parameters of the probability distributions describing the aleatory uncertainty are themselves affected by epistemic uncertainty and represented in terms of possibility distributions.

\section{The yearly maximal water flow, $Y_{1}=Q$}

The aleatory uncertainty in the yearly maximal water flow $Y_{1}=Q$ is well described by a Gumbel probability distribution $p^{Q}(q \mid \gamma, \delta)=\operatorname{Gum}(\gamma, \delta)=\frac{1}{\delta} \exp \left[-\exp \left(\frac{\gamma-q}{\delta}\right)\right] \exp \left[\frac{\gamma-q}{\delta}\right][$ Limbourg and de Rocquigny, 2010]. The extreme physical bounds on variable $Q$ are [Limbourg and de Rocquigny, 2010]:

- $\quad Q_{\min }=10 \mathrm{~m}^{3} / \mathrm{s}$, which is a typical drought flow level (irrelevant within a flood study); - $Q_{\max }=10000 \mathrm{~m}^{3} / \mathrm{s}$, which is three times larger than the maximal flood ever occurred.

When Approach A is used, the prior possibility distributions $\pi^{\gamma}(\gamma)$ and $\pi^{\delta}(\delta)$ for the epistemically-uncertain parameters $\gamma$ and $\delta$ are subjectively chosen as triangular functions $\operatorname{TR}\left(a_{\gamma}\right.$, $\left.c_{\gamma}, b_{\gamma}\right)$ and $\operatorname{TR}\left(a_{\delta}, c_{\delta}, b_{\delta}\right)$, respectively, with cores (i.e., preferred or most likely values) $c_{\gamma}=955 \mathrm{~m}^{3} / \mathrm{s}$ and $c_{\delta}=600 \mathrm{~m}^{3} / \mathrm{s}$, and supports $\left[a_{\gamma}, b_{\gamma}\right]=[869,1157] \mathrm{m}^{3} / \mathrm{s}$ and $\left[a_{\delta}, b_{\delta}\right]=[455,660] \mathrm{m}^{3} / \mathrm{s}$, 
421 respectively. When Approach B is employed, the fictitious prior FPDFs $\tilde{p}^{\gamma}(\gamma)$ and $\tilde{p}^{\delta}(\delta)$ are 422 subjectively chosen as normal probability distributions $p^{\gamma}(\gamma)=N\left(\mu_{\gamma}, \sigma_{\gamma}\right)$ and $p^{\delta}(\delta)=$ $423 N\left(\mu_{\delta}, \sigma_{\delta}\right)$, respectively, with possibilistic parameters $\mu_{\gamma}, \sigma_{\gamma}, \mu_{\delta}$ and $\sigma_{\delta}:$ the characteristics of the 424 corresponding triangular possibility distributions $\pi^{\mu_{\gamma}}\left(\mu_{\gamma}\right), \pi^{\sigma_{\gamma}}\left(\sigma_{\gamma}\right), \pi^{\mu_{\delta}}\left(\mu_{\delta}\right)$ and $\pi^{\sigma_{\delta}}\left(\sigma_{\delta}\right)$ are 425 summarized in Table 2 for brevity. The Bayesian update of these uncertainty representations (based 426 on prior subjective knowledge) is realized with the aid of a vector $\boldsymbol{y}_{1}=\left[y_{11}, y_{12}, \ldots, y_{1 k}, \ldots, y_{1 D_{1}}\right]$ of $427 D_{1}=149$ (independent and identically distributed - iid) values of the annual maximal flow of the 428 river, i.e., $\boldsymbol{y}_{1}=\boldsymbol{q}=\left[q_{1}, q_{2}, \ldots, q_{k}, \ldots, q_{149}\right]$. The point estimates for $\gamma$ and $\delta$ obtained by the 429 classical, purely probabilistic Maximum Likelihood Estimation (MLE) method are $\hat{\gamma}^{M L E}=1013.21$ $430 \mathrm{~m}^{3} / \mathrm{s}$ and $\hat{\delta}^{M L E}=558.21 \mathrm{~m}^{3} / \mathrm{s}$, respectively.

\section{The upstream riverbed level, $\boldsymbol{Y}_{2}=Z_{m}$}

432 The aleatory part of the uncertainty in the upstream riverbed level $Y_{2}=Z_{m}$ is represented by a 433 normal distribution, i.e., $Z_{m} \sim p^{Z_{m}}\left(z_{m} \mid \mu_{Z m}, \sigma_{Z m}\right)=N\left(\mu_{Z m}, \sigma_{Z m}\right)$ [Limbourg and de Rocquigny, 434 2010]. This distribution is truncated at the minimum and maximum physical bounds on $Z_{m}$, i.e., $435 Z_{m, \text { min }}=53.5 \mathrm{~m}$ (given by plausible lower geomorphologic limits to erosion) and $Z_{m, \max }=57 \mathrm{~m}$ 436 (given by plausible upper geomorphologic limits to sedimentation), respectively. In Approach A, 437 the prior possibility distributions $\pi^{\mu_{Z m}}\left(\mu_{Z m}\right)$ and $\pi^{\sigma_{Z n}}\left(\sigma_{Z m}\right)$ for $\mu_{Z m}$ and $\sigma_{Z m}$ are chosen as 438 triangular functions (Table 2). In Approach B, the fictitious prior FPDFs $\tilde{p}^{\mu_{Z n}}\left(\mu_{Z m}\right)$ and $\tilde{p}^{\sigma_{Z n}}\left(\sigma_{Z m}\right)$ 439 are chosen as normal PDFs $p^{\mu_{Z m}}\left(\mu_{Z m}\right)=N\left(\mu_{\mu_{Z m}}, \sigma_{\mu_{Z m}}\right)$ and $p^{\sigma_{Z n}}\left(\sigma_{Z m}\right)=N\left(\mu_{\sigma_{Z m}}, \sigma_{\sigma_{Z m}}\right)$ with 440 parameters described by the triangular possibilistic functions $\pi^{\mu_{\mu_{Z m}}}\left(\mu_{\mu_{Z m}}\right), \quad \pi^{\sigma_{\mu Z m}}\left(\sigma_{\mu_{Z m}}\right)$, $441 \pi^{\mu_{\sigma_{Z m}}}\left(\mu_{\sigma_{Z m}}\right)$ and $\pi^{\sigma_{\sigma_{Z m}}}\left(\sigma_{\sigma_{Z m}}\right)$ reported in Table 2. The Bayesian update of these uncertainty representations is carried out using a vector $\boldsymbol{y}_{2}=\left[y_{21}, y_{22}, \ldots, y_{2 k}, \ldots, y_{2 D_{2}}\right]$ of $D_{2}=29$ (iid) values 
443 of the upstream riverbed level, i.e., $\boldsymbol{y}_{2}=\boldsymbol{z}_{\boldsymbol{m}}=\left[z_{m, 1}, z_{m, 2}, \ldots, z_{m, k}, \ldots, z_{m, 29}\right]$. The point estimates $\hat{\mu}_{\mathrm{Zm}}^{M L E}$ 444 and $\hat{\sigma}_{Z m}^{M L E}$ for $\mu_{Z m}$ and $\sigma_{Z m}$ obtained by the MLE method are $\hat{\mu}_{Z m}^{M L E}=50.19 \mathrm{~m}$ and $\hat{\sigma}_{Z m}^{M L E}=0.38 \mathrm{~m}$, 445 respectively.

446 The downstream riverbed level, $Y_{3}=Z_{v}$

447 As for $Y_{2}=Z_{m}$, the aleatory part of the uncertainty in the downstream riverbed level $Y_{3}=Z_{v}$ is 448 represented by a normal distribution, i.e., $Z_{v} \sim p^{Z_{v}}\left(z_{v} \mid \mu_{Z v}, \sigma_{Z v}\right)=N\left(\mu_{Z v}, \sigma_{Z v}\right)$, truncated at $449 Z_{v, \min }=48 \mathrm{~m}$ and $Z_{v, \max }=51 \mathrm{~m}$. As before, the prior possibility distributions $\pi^{\mu_{Z v}}\left(\mu_{Z v}\right)$ and $450 \pi^{\sigma_{Z v}}\left(\sigma_{Z v}\right)$ used in Approach A are triangular functions (Table 2) and the FPDFs $\tilde{p}^{\mu_{Z v}}\left(\mu_{Z v}\right)$ and $451 \quad \tilde{p}^{\sigma_{Z v}}\left(\sigma_{Z v}\right)$ employed in Approach B are normal PDFs $p^{\mu_{Z v}}\left(\mu_{Z v}\right)=N\left(\mu_{\mu_{Z n}}, \sigma_{\mu_{Z m}}\right)$ and $p^{\sigma_{Z v}}\left(\sigma_{Z v}\right)=$ $452 N\left(\mu_{\sigma_{Z v}}, \sigma_{\sigma_{Z v}}\right)$ with parameters described by the triangular possibility distributions $\pi^{\mu_{\mu_{\nu v}}}\left(\mu_{\mu_{Z v}}\right)$, $453 \pi^{\sigma_{\mu \mathrm{Zv}}}\left(\sigma_{\mu_{\mathrm{Zv}}}\right), \pi^{\mu_{\sigma_{Z v}}}\left(\mu_{\sigma_{Z v}}\right)$ and $\pi^{\sigma_{\sigma_{Z v}}}\left(\sigma_{\sigma_{Z v}}\right)$ of Table 2. These representations are updated by means of 454 a vector $\boldsymbol{y}_{3}=\left[y_{31}, y_{32}, \ldots, y_{3 k}, \ldots, y_{3 D_{3}}\right]$ of $D_{3}=29$ (iid) values of the downstream riverbed level, 455 i.e., $\boldsymbol{y}_{3}=z_{v}=\left[z_{v, 1}, z_{v, 2}, \ldots, z_{v, k}, \ldots, z_{v, 29}\right]$. The MLE estimates of the parameters are $\hat{\mu}_{Z v}^{M L E}=55.03 \mathrm{~m}$ 456 and $\hat{\sigma}_{Z v}^{M L E}=0.45 \mathrm{~m}$, respectively.

The Strickler friction coefficient, $Y_{4}=K_{s}$

458 The Strickler friction coefficient $Y_{4}=K_{s}$ is the most critical source of uncertainty because it is usually a simplification of a complex hydraulic model. The absolute physical limits of $K_{s}$ are 5 and 60, respectively [Limbourg and de Rocquigny, 2010]:

$461 \quad-\quad K_{s}<5$ corresponds to an "extremely sinuous shape of the canal, with large dents and strong vegetation";

- $K_{s}=60$ corresponds to a "canal with smoothest earth surface, rectilinear, without any vegetation". 
465 The friction coefficient $K_{s}$ is affected by random events modifying the river status (e.g., erosion, 466 sedimentation, ...): the corresponding variability is typically described by a normal distribution, i.e., $K_{s} \sim p^{K_{s}}\left(k_{s} \mid \mu_{K s}, \sigma_{K s}\right)=N\left(\mu_{K s}, \sigma_{K s}\right)$ [Limbourg and de Rocquigny, 2010]. However, the parameters of this normal distribution are difficult to estimate because data can only be obtained through "indirect calibration characterized by significant uncertainty" [Limbourg and de Rocquigny, 2010]: the uncertainty in these parameters is described by possibility distributions.

Details about the possibilistic functions $\pi^{\mu_{K_{s}}}\left(\mu_{K_{s}}\right)$ and $\pi^{\sigma_{K s}}\left(\sigma_{K s}\right)$ and the FPDFs $\tilde{p}^{\mu_{K s}}\left(\mu_{K s}\right)$ and $\tilde{p}^{\sigma_{K s}}\left(\sigma_{K s}\right)$ used to represent the a priori knowledge on $\mu_{K s}$ and $\sigma_{K s}$ in Approaches $\mathrm{A}$ and B, respectively, are reported in Table 2. The Bayesian revision of these a priori representations is performed by means of a vector $\boldsymbol{y}_{4}=\left[y_{41}, y_{42}, \ldots, y_{4 k}, \ldots, y_{4 D_{4}}\right]$ of $D_{4}=5$ (iid) values of the

Strickler friction coefficient, i.e., $\boldsymbol{y}_{4}=\boldsymbol{k}_{s}=\left[k_{s, 1}, k_{s, 2}, \ldots, k_{s, k}, \ldots, k_{s, 5}\right]$. The MLE estimates of the parameters are $\hat{\mu}_{K s}^{M L E}=27.8$ and $\hat{\sigma}_{K s}^{M L E}=5.26$, respectively.

\section{Table 2.}

\section{Results}

In order to simplify the notation, in what follows let $\theta$ be one of the uncertain parameters of the PDFs of $Y_{1}=Q, Y_{2}=Z_{m}, Y_{3}=Z_{v}$ and $Y_{4}=K_{s}$, i.e., $\theta=\gamma, \delta, \mu_{Z m}, \sigma_{Z m}, \mu_{Z v}, \sigma_{Z v}, \mu_{K_{s}}$ or $\sigma_{K s}$. Fig. 3 illustrates the possibility distributions of the epistemically-uncertain parameters of the aleatory PDFs $p^{Q}(q \mid \gamma, \delta)$ (top row), $p^{Z_{m}}\left(z_{m} \mid \mu_{Z m}, \sigma_{Z m}\right)$ (middle-top row), $p^{Z_{v}}\left(z_{v} \mid \mu_{Z v}, \sigma_{Z v}\right)$ (middle-bottom row) and $p^{K_{s}}\left(k_{s} \mid \mu_{K s}, \sigma_{K s}\right)$ (bottom row) of the uncertain input variables $Y_{1}=Q, Y_{2}=Z_{m}, Y_{3}=Z_{v}$ and $Y_{4}=K_{s}$, respectively, of the model of the previous Section: in particular, the prior possibility distributions $\pi^{\theta}(\theta)\left(=\pi^{\gamma}(\gamma), \pi^{\delta}(\delta), \pi^{\mu_{Z m}}\left(\mu_{Z m}\right), \pi^{\sigma_{Z m}}\left(\sigma_{Z m}\right), \pi^{\mu_{Z v}}\left(\mu_{Z v}\right), \pi^{\sigma_{Z v}}\left(\sigma_{Z v}\right), \pi^{\mu_{K_{s}}}\left(\mu_{K_{s}}\right)\right.$ and $\left.\pi^{\sigma_{K s}}\left(\sigma_{K s}\right)\right)$ are shown as solid lines, whereas the marginal posterior possibility distributions 
$\pi^{\theta}(\theta \mid \boldsymbol{y}) \quad\left(=\pi^{\gamma}(\gamma \mid \boldsymbol{q}), \pi^{\delta}(\boldsymbol{\delta} \mid \boldsymbol{q}), \pi^{\mu_{Z m}}\left(\mu_{Z m} \mid z_{m}\right), \pi^{\sigma_{Z m}}\left(\sigma_{Z m} \mid z_{m}\right), \pi^{\mu_{Z v}}\left(\mu_{Z v} \mid z_{v}\right), \pi^{\sigma_{Z v}}\left(\sigma_{Z v} \mid z_{v}\right)\right.$, $\pi^{\mu_{K_{s}}}\left(\mu_{K_{s}} \mid \boldsymbol{k}_{s}\right)$ and $\left.\pi^{\sigma_{K s}}\left(\sigma_{K s} \mid \boldsymbol{k}_{s}\right)\right)$ obtained by Approaches A and B using $D_{1}=149, D_{2}=29, D_{3}=$ 29 and $D_{4}=5$ pieces of data are shown in dashed and dot-dashed lines, respectively; the point estimates $\hat{\theta}^{M L E}\left(=\hat{\gamma}^{M L E}, \hat{\delta}^{M L E}, \hat{\mu}_{Z m}^{M L E}, \hat{\sigma}_{Z m}^{M L E}, \hat{\mu}_{Z v}^{M L E}, \hat{\sigma}_{Z v}^{M L E}, \hat{\mu}_{K_{s}}^{M L E}\right.$ and $\left.\hat{\sigma}_{K s}^{M L E}\right)$ produced by the classical MLE method are also shown for comparison (dots).

Fig. 3.

From a mere visual and qualitative inspection of Fig. 3 it can be seen that both approaches are suitable for revising the prior possibility distributions (based on a priori purely subjective knowledge) by means of empirical data. In particular, it is evident that: (i) the most likely (i.e., preferred) values $c_{\theta}$ of the epistemically-uncertain parameters (i.e., those values in correspondence of which the possibility function equals 1 ) are moved towards the MLE estimates $\hat{\theta}^{M L E}$ in all the cases considered; (ii) the area $S_{\theta}$ underlying the corresponding possibility distributions is significantly reduced: noting that this area is related to the imprecision in the knowledge of the possibilistic parameter (i.e., the larger the area, the higher the imprecision), it can be concluded that both approaches succeed in reducing the epistemic uncertainty. With respect to that, Table 3 reports the most likely values $c_{\theta}$ and the areas $S_{\theta}$ underlying the (marginal) possibility distributions of the uncertain parameter $\theta\left(=\gamma, \delta, \mu_{Z m}, \sigma_{Z m}, \mu_{Z v}, \sigma_{Z v}, \mu_{K_{s}}\right.$ and $\left.\sigma_{K s}\right)$ before and after the Bayesian update performed by Approaches A and B; the point estimates $\hat{\theta}^{M L E}$ obtained by the classical MLE method are also reported for completeness. In addition, in order to perform a quantitative comparison between the methods, two indicators are defined:

511 i. the relative absolute distance $d_{\theta}^{M L E}$ between the (posterior) most likely value $c_{\theta}$ of parameter $\theta$ and the corresponding MLE estimate $\hat{\theta}^{M L E}$, i.e.: 


$$
d_{\theta}^{M L E}=\frac{\left|c_{\theta}-\hat{\theta}^{M L E}\right|}{\hat{\theta}^{M L E}} .
$$

Obviously, the lower is $d_{\theta}^{M L E}$ the closer is the most likely value $c_{\theta}$ to the MLE estimate $\hat{\theta}^{M L E}$, i.e., the higher is the strength of the approach in updating the prior possibilistic distribution on the basis of newly available experimental evidence;

ii. the percentage relative difference $R_{\theta}$ between the areas underlying the possibility

B for all the parameters.

From the analysis of quantitative indicator $R_{\theta}(6)$ it can be seen that both methods succeed in reducing the area underlying the possibility distributions of the uncertain parameters: in particular, the percentage reduction $R_{\theta}$ ranges between $8.33 \%$ and $30.49 \%$ for approach $\mathrm{A}$ and between $3.89 \%$ and $33.01 \%$ for Approach B. In addition, as expected, the strength of both approaches in reducing 535 For example, the area $S_{\gamma}$ underlying the possibility distribution of $\gamma$ (Fig. 3, top left column) is 536 reduced by $30.49-33.01 \%$ with the aid of a large data set of size $D_{1}=149$; on the contrary, the area 
$S_{\sigma_{K s}}$ underlying the possibility distribution of $\sigma_{K s}$ (Fig. 3, bottom right column) is reduced only by

$3.89-8.76 \%$ by means of $D_{4}=5$ pieces of data. Moreover, it is interesting to note that the strength of Approach B in reducing epistemic uncertainty is slightly higher than that of Approach A only when the amount of available data is quite large (i.e., in the revision of the possibility distributions of parameters $\gamma$ and $\delta$ of the PDF of $Y_{1}=Q$, by means of $D_{1}=149$ pieces of data): actually, the values of $R_{\gamma}$ and $R_{\delta}$ range within $25.56-30.49 \%$ and $28.74-33.01 \%$ for Approaches A and B, respectively. In all the other cases, the power of Approach A in reducing epistemic uncertainty is higher than that of Approach B and this difference becomes more and more evident as the size of the data set decreases: in particular, for medium-sized data sets (i.e., in the revision of the possibility distributions of the parameters $\mu_{Z m}, \sigma_{Z m}, \mu_{Z v}, \sigma_{Z v}$ of the PDFs of $Y_{2}=Z_{m}$ and $Y_{3}=Z_{v}$ with $D_{2}=D_{3}=29$ pieces of data) the values of $R_{\theta}(6)$ produced by Approaches A and B range within $8.33-24.38 \%$ and $4.17-22.00 \%$, respectively; instead, for small-sized data sets (i.e., in the revision of the possibility distributions of the parameters $\mu_{K_{s}}$ and $\sigma_{K s}$ of the PDF of $Y_{4}=K_{s}$ with $D_{4}=5$ pieces of data) the values of $R_{\theta}$ produced by Approaches A and B range within 8.76-16.28\% and $3.89-10.08 \%$, respectively. This is particularly evident in the estimation of the standard deviation $\sigma_{K s}$ of $K_{s}$ (Fig. 3, bottom right column): on one side, the posterior distribution produced by the hybrid approach (B) seems not to be influenced by the revision process (actually, the most likely value of the parameter, $c_{\sigma_{K s}}=6.72$, and the area underlying the corresponding posterior possibility distribution, $S_{\sigma_{K s}}=3.95$, are quite close to those of the prior, i.e., 6.89 and 4.11, respectively); on the other side, the posterior distribution generated by the purely possibilistic approach (A) is almost centered on the point estimates obtained by the MLE method and the corresponding area is reduced by about $9 \%$.

Finally, it has to be remarked that for the sake of simplicity the quantitative analyses above have 561 been performed only on the marginal posterior possibility distributions of the uncertain parameters 
$\left(\pi^{\gamma}(\gamma \mid \boldsymbol{q}), \pi^{\delta}(\delta \mid \boldsymbol{q}), \pi^{\mu_{Z m}}\left(\mu_{Z m} \mid z_{m}\right), \pi^{\sigma_{Z n}}\left(\sigma_{Z m} \mid z_{m}\right), \pi^{\mu_{Z v}}\left(\mu_{Z v} \mid z_{v}\right), \pi^{\sigma_{Z v}}\left(\sigma_{Z v} \mid z_{v}\right), \pi^{\mu_{K_{s}}}\left(\mu_{K_{s}} \mid \boldsymbol{k}_{s}\right)\right.$

and $\left.\pi^{\sigma_{K s}}\left(\sigma_{K s} \mid \boldsymbol{k}_{s}\right)\right)$; in fact, as highlighted before, the posterior possibility distributions of the uncertain parameters are multi-dimensional functions (and the posterior estimates of the parameters are dependent). Only for illustration purposes, Fig. 4 shows the $\alpha$-cuts of level $\alpha=0.05,0.50$ and 0.95 of the joint posterior possibility distributions $\pi^{\gamma, \delta}(\gamma, \delta \mid \boldsymbol{q}), \quad \pi^{\mu_{Z m}, \sigma_{Z m}}\left(\mu_{Z m}, \sigma_{Z m} \mid z_{m}\right)$, $\pi^{\mu_{Z v}, \sigma_{Z v}}\left(\mu_{Z v}, \sigma_{Z v} \mid z_{v}\right)$ and $\pi^{\mu_{K_{s}}, \sigma_{K_{s}}}\left(\mu_{K_{s}}, \sigma_{K_{s}} \mid \boldsymbol{k}_{s}\right)$ of the parameters of the PDFs of $Y_{1}=Q$ (top right column), $Y_{2}=Z_{m}$ (top left column), $Y_{3}=Z_{v}$ (bottom left column) and $Y_{4}=K_{s}$ (bottom right column), respectively, produced by Approaches A (solid lines) and B (dashed lines).

\section{Fig. 4.}

The visual inspection of Fig. 4 confirms the results obtained by the quantitative analysis carried out on the marginal distributions. For example, it can be seen that the areas of the $\alpha$-cuts of level $\alpha=$ 0.95 of $\pi^{\mu_{z v}, \sigma_{Z v}}\left(\mu_{Z v}, \sigma_{Z v} \mid z_{v}\right)$ and $\pi^{\mu_{K_{s}}, \sigma_{K_{s}}}\left(\mu_{K_{s}}, \sigma_{K_{s}} \mid \boldsymbol{k}_{s}\right)$ produced by Approach A are consistently

smaller than those generated by Approach B; the $\alpha$-cuts of $\pi^{\mu_{Z m}, \sigma_{Z m}}\left(\mu_{Z m}, \sigma_{Z m} \mid z_{m}\right)$ produced by Approaches A and B are comparable in size, whereas the area of the $\alpha$-cut of $\pi^{\gamma, \delta}(\gamma, \delta \mid \boldsymbol{q})$ obtained by Approach B is slightly lower than the one produced by Approach A.

In order to show the effect that the reduction of the epistemic uncertainty in the distribution parameters has on the uncertain input variables $Y_{1}=Q, Y_{2}=Z_{m}, Y_{3}=Z_{v}$ and $Y_{4}=K_{s}$, Fig. 5 reports the upper and lower CDFs, $\bar{F}^{Y_{j}}\left(y_{j}\right)$ and $\underline{F}^{Y_{j}}\left(y_{j}\right)$ (i.e., the plausibility and belief functions, $\operatorname{Pl}^{Y_{j}}\left(\left(-\infty, y_{j}\right]\right)$ and $\operatorname{Bel}^{Y_{j}}\left(\left(-\infty, y_{j}\right]\right)$, respectively), $j=1,2,3,4$, of $Y_{1}=Q$ (top left column), $Y_{2}=Z_{m}$ (top right column), $Y_{3}=Z_{v}$ (bottom left column) and $Y_{4}=K_{s}$ (bottom right column) before (solid lines) and after the Bayesian update performed by Approaches A (dashed lines) and B (dot-dashed 
lines). The calculation of CDFs is of great importance in risk assessment since they summarize the uncertainty "contained" in the variables of interest.

Obviously, the gap between the plausibility and belief functions $P l^{Y_{j}}\left(\left(-\infty, y_{j}\right]\right)=\bar{F}^{Y_{j}}\left(y_{j}\right)$ and $\operatorname{Bel}^{Y_{j}}\left(\left(-\infty, y_{j}\right]\right)=\underline{F}^{Y_{j}}\left(y_{j}\right), j=1,2,3,4$, is larger before the Bayesian update in all the cases considered: in particular, the 'prior' CDFs (solid lines) completely envelop the 'posterior' ones (dashed and dot-dashed lines). This larger gap is explained by the larger area contained under the possibility distribution functions of the corresponding epistemically-uncertain parameters (actually, as highlighted before, the larger the area, the higher the imprecision in the knowledge of the possibilistic parameters). Then, in order to provide a fair and quantitative comparison between the two approaches adopted,

599 the intervals $\left[\left[\bar{F}^{Y_{j}}\right]^{-1}(\beta),\left[\underline{F}^{Y_{j}}\right]^{-1}(\beta)\right]$ for the $\beta \cdot 100$-th percentiles $Y_{j}^{\beta}, \beta=0.05,0.50$ and 0.95 , of the 600 variables $Y_{j}, j=1,2,3,4$, are computed (Table 4). For example, analyzing $Q^{\beta}, \beta=0.05,0.50$ and 0.95 , it can be seen that the width of the intervals is reduced by $30.54-40.34 \%$ and $29.88-43.29 \%$ by Approaches A and B, respectively: coherently with the results reported in Table 3, when a large data set is available (i.e., $D_{1}=149$ in this case) the strength of Approach $\mathrm{B}$ in reducing the epistemic uncertainty is slightly (i.e., 2-3\%) higher than that of Approach A. On the contrary, 25.30-30.80\% and 5.74-19.45\% by Approaches A and B, respectively: as highlighted before (Table 3), when a small data set is available (i.e., $D_{4}=5$ in this case) the power of Approach A in reducing epistemic uncertainty is consistently (i.e., 15-20\%) higher than that of Approach B. This is confirmed also by the analysis of the quantiles of $Y_{2}=Z_{m}$ and $Y_{3}=Z_{v}$ (see Table 4). 
613 Comparable conclusions can be drawn by the analysis of the upper and lower CDFs $\bar{F}^{z_{c}}\left(z_{c}\right)$ and $614 \underline{F}^{Z_{c}}\left(z_{c}\right)$ (i.e., the plausibility and belief functions, $P l^{Z_{c}}\left(\left(-\infty, z_{c}\right]\right)$ and $B e l^{Z_{c}}\left(\left(-\infty, z_{c}\right]\right)$, 615 respectively), of the maximal water level of the river $Z_{c}$ (i.e., the model output) obtained before 616 (Fig. 6, solid lines) and after the Bayesian update performed by Approaches A (Fig. 6, dashed lines) 617 and B (Fig. 6, dot-dashed lines). Notice that the distributions for $Z_{c}$ have been obtained by 618 propagating the two-level mixed probabilistic and possibilistic uncertainty through the 619 mathematical model by means of a hybrid Monte Carlo (MC) and Fuzzy Interval Analysis (FIA) 620 approach. This method combines the MC technique [Kalos and Withlock, 1986] with the extension 621 principle of fuzzy set theory [Guyonnet et al., 2003; Kentel and Aral, 2004 and 2007] in two 622 hierarchical, repeated steps [Baudrit et al., 2008; Kentel and Aral, 2005; Moller and Beer, 2004 and 623 2008; Moller et al., 2003 and 2006; Pedroni and Zio, 2012; Pedroni et al., 2013]: the reader is 624 referred to the cited references for details. Again, in order to provide a fair comparison between the 625 two approaches employed, proper quantitative indicators are computed. The final goal of the case 626 study presented in the previous Section is to determine (i) the dike level necessary to guarantee a given flood return period or (ii) the flood risk for a given dike level. With respect to issue (i) above, 628 the quantity of interest that is most relevant to the decision maker is the $\beta \cdot 100 \%$-th quantile of $Z_{c}$ 629 (i.e., $Z_{c}^{\beta}$ ): this corresponds to the yearly maximal water level with a $\beta \cdot 100$-year return period. With 630 respect to issue (ii) above, the quantity of interest that is most relevant to the decision maker is the 631 probability that the maximal water level of the river $Z_{c}$ exceeds a given threshold $z^{*}$, i.e., $632 P\left[Z_{c} \geq z_{c}^{*}\right]$ : in the present paper, $z_{c}{ }^{*}=55.5 \mathrm{~m}$ (Table 5). Analyzing the intervals $\left[\left[\bar{F}^{Z_{c}}\right]^{-1}(\beta)\right.$, $\left.633\left[\underline{F}^{Z_{c}}\right]^{-1}(\beta)\right]$ for the percentiles $Z_{c}^{\beta}, \quad \beta=0.05, \quad 0.50$ and 0.95, and the intervals $634\left[1-\bar{F}^{Z_{c}}\left(z_{c}^{*}\right), 1-\underline{F}^{Z_{c}}\left(z_{c}^{*}\right)\right]$ for the exceedance probability $P\left[Z_{c}>z_{c}^{*}\right]$, it can be seen that their width is 635 reduced of $28.14-38.63 \%$ and $16.88-29.23 \%$ by Approaches $\mathrm{A}$ and B, respectively (i.e., the 
strength of Approach A in reducing epistemic uncertainty is about 4-11\% higher than that of 637 Approach B).

\section{Table 5.}

Several considerations are in order with respect to the results obtained. When the Bayesian update is performed based on a data set of large size (e.g., > 100 in this case), the difference in the behavior of the two approaches is quite low. This demonstrates that although the two methods are conceptually and algorithmically quite different, in presence of a "strong" experimental evidence they produce "coherent" results (i.e., posterior possibility distributions that bear the same overall "uncertainty content"): this is a fair outcome since the results provided by the two methods are expected to be more and more similar (i.e., more and more coherent with the experimental evidence) as the size of the data set increases (experts and practitioners may find a similarity and parallelism between these results and those obtained in purely probabilistic, graphical Bayesian models [Gelman et al., 2004]; in particular, see references concerning approaches used to "borrow strength" in (Hierarchical) Bayesian analyses [Atwood et al., 2003; Kelly and Smith, 2009 and 2011]). Instead, when the Bayesian update is performed on a data set of small-medium size (e.g., $\approx$ 5-30 in this case), the strength of Approach A in reducing epistemic uncertainty is significantly possibilistic likelihood (i.e., the function "containing" the experimental evidence available) has a 658 direct and strong influence on the purely possibilistic prior (actually, they are directly multiplied in 659 (1)); on the contrary, in Approach B the purely probabilistic likelihood has a direct influence only 660 on the (fictitious) probabilistic distributions that are superimposed onto the prior possibilistic 661 parameters: this "artificial" procedure may in practice "soften" (i.e., reduce) the effect of the newly 662 available information (i.e., of the data) in the revision of the possibilistic priors (obviously, this 
663 effect is expected to be more evident if the amount of data, i.e., the strength of the experimental 664 evidence, is small). In such cases, embracing one method instead of the other may significantly 665 change the outcome of a decision making process in a risk assessment problem involving 666 uncertainties: this is of paramount importance in systems that are critical from the safety view point, 667 e.g., in the nuclear, aerospace, chemical and environmental fields.

668 Finally, it is absolutely important to acknowledge that even if the strength of one method (i.e., A in 669 this case) in reducing epistemic uncertainty is higher than that of the other one (i.e., B in this case), 670 this does not necessarily imply that one method is "better" or "more effective" than the other 671 overall. Actually, if on one side a consistent reduction in the epistemic uncertainty is in general 672 desirable in decision making processes related to risk assessment problems (since it significantly 673 increases the analyst's confidence in the decisions), on the other side this reduction must be coherent with the amount of information available. In this view, an objection may arise in the present case: is the remarkable strength of Approach A in reducing epistemic uncertainty (with very few pieces of data) fully justified by such a small amount of data? In other words, is this considerable reduction of epistemic uncertainty coherent with the strength of the experimental evidence or is it too optimistic? These issues will be thoroughly discussed in the following dedicated Section.

Finally, in addition to the strength of the approaches in revising the (prior) possibilistic description of the uncertain parameters of aleatory variables, also the computational time associated to the methods has to be taken into account. Table 6 reports the computational time $t_{\text {comp }}$ required by the Bayesian update of all the parameters of the PDFs of $Y_{1}=Q, Y_{2}=Z_{m}, Y_{3}=Z_{v}$ and $Y_{4}=K_{s}$ performed by Approach A and by Approach B (with $T=100$ repetitions of the purely probabilistic Bayes' theorem for each of the $N_{\alpha}=21 \alpha$-cuts analyzed). Obviously, the computational time required by Approach B is approximately $T \cdot N_{\alpha}$ (i.e., $T \cdot N_{\alpha}=100 \cdot 21=2100$ in this case) times larger than that of Approach A. On the other hand, notice that since Approach B is based on several repetitions of the 
purely probabilistic Bayes' theorem, if possible, parallelization could be in principle employed to reduce the associated computational cost.

\section{Table 6.}

\section{Discussion of the results and comparison of the approaches}

The results of the comparisons performed in the previous Sections can be summarized as follows:

- both methods succeed in updating the possibilistic description of the epistemically-uncertain parameters of (aleatory) probability distributions by means of data. This is highlighted by the fact that in most cases the posterior possibility distributions produced by the two approaches are significantly different from the corresponding priors. In particular:

- the most likely values of the parameters (i.e., those values in correspondence of which the possibility function equals 1) are moved towards the point estimates of the parameters obtained by the classical, purely probabilistic MLE method;

- the area underlying the posterior possibility distributions is consistently lower than that of the priors: since this area is related to the imprecision in the knowledge of the possibilistic parameter (i.e., the larger the area, the higher the imprecision), it can be concluded that both approaches succeed in reducing the epistemic uncertainty in the possibilistic parameters of the aleatory probability distributions. This is also confirmed by the reduction of the gap between the upper and lower CDFs (i.e., the plausibility and belief functions) of the corresponding aleatory variables;

- when the Bayesian update is performed using a data set of large size (e.g., > 100), the strength of the two approaches in reducing the epistemic uncertainty is quite similar. By way of example, in the case study considered Approaches A and B reduce the areas underlying the possibility distributions of the uncertain parameters by $25.56-30.49 \%$ and 28.74-33.01\%, respectively. This demonstrates that although the two methods are 
conceptually and algorithmically quite different, in presence of a "strong" experimental evidence they produce "coherent" results (i.e., possibility distributions that bear almost the same overall "uncertainty content"): this is a fair outcome since the it is "desired" that both methods provide results that become more and more similar (i.e., that become more and more coherent with the experimental evidence) as the size of the data set increases;

- the strength of the purely possibilistic approach (A) in reducing epistemic uncertainty is consistently higher than that of the hybrid one (B) in presence of medium- and small-sized data sets (e.g., $\approx 5-30$ ) (which is often the case in the risk analysis of complex safety-critical systems). For example, the width of the intervals for the quantiles of the variables of interest is reduced by $25.30-30.80 \%$ and $5.74-19.45 \%$ by Approaches $\mathrm{A}$ and $\mathrm{B}$, respectively. This significantly different behavior is explained by the fact that in Approach A the (purely possibilistic) likelihood has an immediate and strong influence on the (purely possibilistic) prior (i.e., they are directly multiplied); on the contrary, in Approach B the (purely probabilistic) likelihood has a direct influence only on the (fictitious) probabilistic function that is superimposed onto the possibilistic parameter (subject to the Bayesian update): this "artificial" procedure could in practice weaken the effect of the newly available information (i.e., the data) in the revision of the possibilistic prior (this effect is expected to be more evident if the amount of data, i.e., the strength of the experimental evidence, is small).

As highlighted above, the fact that the power of Approach A in reducing epistemic uncertainty is higher than that of Approach B does not necessarily imply that one method is "better" or "more effective" than the other overall. For example, by hypothesis the remarkable strength of Approach A in reducing epistemic uncertainty by means of very few pieces of data (e.g., 5-30 in this case) might not be coherent with the "real" strength of such a scarce experimental evidence and could be accidentally due to some bias in the procedure. With respect to that, it has to be admitted that the uncertainty reduction power of the purely possibilistic approach (A) is strongly dependent on the shape of a constructed possibilistic 
likelihood that could in principle bias the analysis. However: (i) in the present paper, this possibilistic function is very closely related to the classical, purely probabilistic one (which is theoretically well-grounded) by a simple and direct operation of normalization that preserves the "original structure" of the experimental evidence; (ii) the operation of normalization of the probabilistic likelihood finds also some theoretical justification in the work by [Denoeux, 2014]; (iii) in general, a probability-to-possibility transformation (properly performed according to the rules of possibility theory) always introduces additional artificial epistemic uncertainty into the analysis, i.e., it does not artificially reduce it (because it replaces a single probabilistic distribution by a family of distributions) [Dubois et al., 1993, 2004 and 2008; Flage et al., 2010 and 2013]. On the basis of considerations (i)(iii) above, it seems unlikely that the purely possibilistic approach (A) may produce results that are dangerously over-optimistic with respect to those of the hybrid one (B). On the other hand, future research should be devoted to the study and development of rigorous, generalized methods for Bayesian model comparison and validation in a purely possibilistic metrics originally introduced in the present manuscript: in this light, techniques from the classical, purely probabilistic field may serve as inspiring references [Gelman et al., 1996; Bayarri and Berger, 1999 and 2000; Johnson Valen, 2004];

- the computational time required by the hybrid approach (B) is consistently higher than that associated to the purely possibilistic one (A): this is explained by the necessity of repeatedly applying many times the purely probabilistic Bayes' theorem for each $\alpha$-cut analyzed. More precisely, the application of method A just requires one single evaluation of the purely possibilistic Bayes' formula; on the other hand, approach B entails repeating $T \cdot N_{\alpha}$ times the classical probabilistic Bayes' theorem. In this respect, notice that $N_{\alpha}$ (i.e., the number of $\alpha$ cuts processed) is typically of the order of 10 [Baudrit et al., 2006], whereas $T$ (i.e., the number of parameter values selected to explore each $\alpha$-cut) cannot be in principle prescribed 
a priori: however, it needs to be large enough to thoroughly explore each dimension of the epistemic parameter space (at least 10 values should be selected for each parameter);

- both methods relies on assumptions about either the prior or the likelihood functions: in the

\section{Conclusions}

783 In this paper, we have considered two methods for the Bayesian update of the possibilistic parameters of aleatory probability distributions, with exemplification on a case study concerning the risk-based design of a flood protection dike. The first method considered is based on a purely possibilistic counterpart of the classical probabilistic Bayes' theorem; the second is a hybrid 787 (probabilistic and possibilistic) method combining Fuzzy Interval Analysis and the classical 788 probabilistic Bayes' theorem.

789 The findings of the work show that in general adopting different methods may generate different 790 results and possibly different decisions in risk problems involving uncertainties: this is of 791 paramount importance in systems that are critical from the safety viewpoint, e.g., in the nuclear, 792 aerospace, chemical and environmental fields. 
793 In particular, on the basis of the results obtained, it seems advisable to suggest the use of the purely 794 possibilistic approach (instead of the hybrid one) for the following reasons: (i) its strength in 795 reducing epistemic uncertainty is significantly higher, in particular when the amount of available 796 data is small: this is important in decision making processes since reducing epistemic uncertainty 797 significantly increases the analyst confidence in the decisions; (ii) the computational time required 798 is consistently lower.

799 However, it has to be remarked that the construction of a possibilistic likelihood required by the 800 purely possibilistic method, although recently tacked in the literature, still represents an issue to be 801 further investigated from both the theoretical and practical viewpoint in order to avoid introducing 802 biases in the analysis and to suggest the application of the approach for real risk assessment 803 problems: with respect to that, future research will be devoted to the investigation of additional 804 methods developed to this aim. Also, future studies will be aimed at developing generalized 805 methods for Bayesian model comparison and validation in a purely possibilistic framework, in order 806 to complement and strengthen the conclusions drawn by means of the metrics originally introduced 807 in the present manuscript. 


\section{References}

811 Anoop MB, Balaji Rao K, Gopalakrishnan S, 2006. Conversion of probabilistic information into fuzzy sets for engineering decision analysis. Computers and Structures, 84, pp. 141-155.

Apostolakis, G.E., 1990. The concept of probability in safety assessment of technological systems. Science, 250, 1359.

Apostolakis, G.E., S. Kaplan, 1981. Pitfalls in risk calculations. Reliability Engineering, Vol. 2, Issue 2, pp. 135-145.

Atwood, C.L., JL. LaChance, H.F. Martz, D.L Anderson, M. Englehardte, D. Whitehead, T. Wheeler, 2003. Handbook of Parameter Estimation for Probabilistic Risk Assessment. NUREG/CR-6823, US Nuclear Regulatory Commission, Washington, DC; SAND20033348P, Sandia National Laboratories, Albuquerque, New Mexico.

Aven, T., 2010. On the Need for Restricting the Probabilistic Analysis in Risk Assessments to Variability. Risk Analysis, Vol. 30, Issue 3, pp. 354-360.

Aven, T., 2011. Interpretations of alternative uncertainty representations in a reliability and risk analysis context. Reliability Engineering \& System Safety, Volume 96, Issue 3, pp. 353-360.

Aven, E. Zio, 2011. Some considerations on the treatment of uncertainties in risk assessment for practical decision making, Reliability Engineering and System Safety, Vol. 96, Issue 1, pp. 64-74.

Ballesteros, G., Angelikopoulos, P., Papadimitriou, C., Koumoutsakos, P., 2014. Bayesian Hierarchical Models for Uncertainty Quantification in Structural Dynamics. Vulnerability, Uncertainty, and Risk: Quantification, Mitigation, and Management, ASCE, pp. 1615-1624.

Baudrit, D. Dubois, 2006. Practical Representations of Incomplete Probabilistic Knowledge, Computational Statistics \& Data Analysis, Vol. 51, Issue 1, pp. 86-108. 
Baudrit, I. Couso, D. Dubois, 2007. Joint propagation of probability and possibility in risk analysis: toward a formal framework, Internat. J. Approx. Reasoning, Vol. 45, Issue 1, pp. 82-105.

Baudrit, D. Dubois, D. Guyonnet, 2006. Joint Propagation and Exploitation of Probabilistic and Possibilistic Information in Risk Assessment. IEEE Transactions on Fuzzy Systems, Vol. 14, Issue 5, pp. 593-608.

Baudrit, D. Dubois, N. Perrot, 2008. Representing parametric probabilistic models tainted with imprecision, Fuzzy Sets and System, Vol. 159, Issue 15, pp.1913-1928.

Bayarri MJ, Berger, JO, 1999. Quantifying surprise in the data and model verification. In: Bernardo, JM, et al., editors. Bayesian statistics 6. Oxford: Oxford University Press. p.53-82.

Bayarri, MJ, Berger, JO, 2000. P-values for composite null models. J Am Stat Assoc; 95: 1127-42.

Bedford, T., Cooke, R., 2001. Probabilistic Risk Analysis. Foundations and Methods. Cambridge: Cambridge University Publishing Ltd.

Beer, M., 2009a. Engineering quantification of inconsistent information, Int J Reliability and Safety, 3(1/2/3): 174-197.

Beer, M., 2009b. Fuzzy probability theory, in: Encyclopedia of Complexity and Systems Science, vol.6, Springer, NewYork, pp.4047-4059.

Beer, M., and Ferson, S., 2013. Special issue of Mechanical Systems and Signal Processing "Imprecise probabilities - What can they add to engineering analyses?". Mechanical Systems and Signal Processing, 37(1-2), 1-3.

Beer, M., Ferson, S., and Kreinovich, V., 2013a. Imprecise probabilities in engineering analyses. Mechanical Systems and Signal Processing, 37(1-2), 4-29.

Beer, M., Zhang, Y., Quek, S.T., Phoon, K.K., 2013b. Reliability analysis with scarce information: Comparing alternative approaches in a geotechnical engineering context, Structural Safety, Vol. 41, pp. 1-10. 
Beer, M., DiazDelaO, F.A., Patelli, E., Au, S.K., 2014a. Conceptual comparison of Bayesian approaches and imprecise probabilities. In: Topping, B.H.V.; Ivanyi, P. (eds.), Computational Technology Reviews 9, 2014, 1-29, Saxe-Coburg Publications.

Beer, M., Kougioumtzoglou, I.A., Patelli, E., 2014b. Emerging Concepts and Approaches for Efficient and Realistic Uncertainty Quantification. In: Frangopol, D.M.; Tsompanakis, Y. (eds.), Maintenance and Safety of Aging Infrastructure, Book Series "Structures \& Infrastructures", Vol 10, Chapter 5, 121-154, CRC Press, Taylor \& Francis Group, Boca Raton, London, New York, Leiden.

Behmanesh, I., Moaveni, B., Lombaert, G., Papadimitriou, C., 2015. Hierarchical Bayesian Model Updating for Probabilistic Damage Identification. In: Atamturktur, HS, Moaveni, B., Papadimitriou, C., Schoenherr, T. Model validation and uncertainty quantification, Volume 3, Conference Proceedings of the Society for Experimental Mechanics Series, pp. 55-66.

Bernard, J.M., 2005. An introduction to the imprecise Dirichlet model for multinomial data, Int. J. Approx. Reason. 39, 123-150.

Bernardo, J M and Smith, A F M, 1994. Bayesian Theory. Chichester: Wiley.

Blockley, D., 2013. Analysing uncertainties: Towards comparing Bayesian and interval probabilities. Mechanical Systems and Signal Processing, 37(1-2), 30-42.

Buckley, J.J., 2005. Fuzzy probabilities — new approach and applications, Studies in Fuzziness and Soft Computing, vol.115, Springer, Berlin, Heidelberg.

Chung, Y., Gelman, A., Rabe-Hesketh, S., Liu, J., Dorie, V., 2015. Weakly Informative Prior for Point Estimation of Covariance Matrices in Hierarchical Models. Journal of Educational and Behavioral Statistics, Vol. 40, No. 2, pp. 136-157.

Congdon, P.D., 2010. Applied Bayesian Hierarchical Methods, CRC Press.

Coolen, F. P. A., and Utkin, L. V., 2007. Imprecise probability: a concise overview." Risk, reliability and societal safety: proceedings of the European safety and reliability conference (ESREL), Stavanger, Norway, 1959-66. 
883 Couso, I., Dubois, D., 2009. On the Variability of the Concept of Variance for Fuzzy Random 884 Variables. Fuzzy Systems, IEEE Transactions on, Volume: 17, Issue: 5, pp. 1070 - 1080.

885 Couso, I., Miranda, E., de Cooman, G., 2004. A Possibilistic Interpretation of the Expectation of a 886 Fuzzy Random Variable. In: Soft Methodology and Random Information Systems, Series in 887 Advances in Soft Computing, Volume 26, pp. 133-140, Springer Berlin Heidelberg.

888 Couso, I., S. Montes, P. Gil, 2001. The necessity of the strong alpha-cuts of a fuzzy set, 889 International Journal of Uncertainty, Fuzziness and Knowledge-Based Systems, 9, 249-262.

890 Couso, I., L. Sánchez, 2008. Higher order models for fuzzy random variables, Fuzzy Sets and $891 \quad$ Systems 159, 237-258.

892 Couso, I., L. Sánchez, 2011. Upper and lower probabilities induced by a fuzzy random variable. 893 Fuzzy Sets and Systems, Volume 165, Issue 1, 16 February 2011, Pages 1-23.

894 Crespo, L. G., Kenny, S. P., and Giesy, D. P., 2013. Reliability analysis of polynomial systems 895 subject to p-box uncertainties. Mechanical Systems and Signal Processing, 37(1-2), 121-136.

896 Denoeux, T., 2014. Likelihood-based belief function: Justification and some extensions to low 897 quality data. International Journal of Approximate Reasoning, Volume 55, Issue 7, Pages 1535$898 \quad 1547$.

899 Dubois, D., 2006. Possibility Theory and Statistical Reasoning. Computational Statistics and Data $900 \quad$ Analysis, Vol. 51, pp. 47-69.

901 Dubois, D., L. Foulloy, G. Mauris, H. Prade, 2004. Probability-Possibility Transformation, 902 Triangular Fuzzy Sets, and Probabilistic Inequalities. Reliable Computing, vol. 10, pp. 273903297.

904 Dubois, D., H. Prade, 1980. Fuzzy Sets and Systems: Theory and Applications, Academic Press, $905 \quad$ New York, NY.

906 Dubois, D., Prade, H., 1988. Possibility Theory: An Approach to Computerized Processing of 907 Uncertainty, New York, Plenum Press. 
908 Dubois, D., Prade, H., 1997. Bayesian conditioning in possibility theory. Fuzzy Sets and Systems, 92, pp. 223-240.

Dubois, D, Prade, H \& Sandri, S, 1993. On possibility/probability transformations. In: Lowen, R \& Roubens, M (eds). Fuzzy Logic: State of the Art. Dordrecht: Kluwer Academic Publishers. pp. 103112.

Dubois D., Prade H., Smets P., 2008. A definition of subjective possibility. International Journal of Approximate Reasoning, 2008, Vol. 48, pp. 352-364.

Dubois D., Moral, S., Prade H., 1997. A Semantics for Possibility Theory Based on Likelihoods. Journal of mathematical analysis and applications, 205, pp. 359-380.

Edwards, A.W.F., 1992. Likelihood, expanded edition. The John Hopkins University Press, Baltimore, USA.

Féron, R., 1976. Ensembles aléatoires flous, CR. Acad. Sc. paris - Série A, Vol 282, 903-906.

Ferson, S., 2005. Bayesian methods in risk assessment. Technical Report: www.ramas.com/bayes.pdf.

Ferson, S. and Hajagos, J.G., 2004. Arithmetic with uncertain numbers: rigorous and (often) best possible answers. Reliability Engineering and System Safety, 85, pp. 135-152.

Ferson, V. Kreinovich, L. Ginzburg, K. Sentz, D.S. Myers, 2003. Constructing probability boxes and Dempster-Shafer structures, Sandia National Laboratories, Technical Report SAND20024015, Albuquerque, New Mexico.

Ferson, V. Kreinovich, J. Hajagos, W. Oberkampf and L. Ginzburg, 2007. Experimental Uncertainty Estimation and Statistics for Data Having Interval Uncertainty, Setauket, New York 11733, Technical Report SAND2007-0939.

Ferson, R.B. Nelsen, J. Hajagos, D.J. Berleant, J. Zhang, W.T. Tucker, L.R. Ginzburg, W.L. Oberkampf, 2004. Dependence in probabilistic modeling, Dempster-Shafer theory, and probability bounds analysis, Technical Report SAND2004-3072, Albuquerque, New Mexico. 
933 Ferson, W.T. Tucker, 2006. Sensitivity in risk analyses with uncertain numbers, Setauket, New York 11733, Technical Report SAND2006-2801.

Ferson, P. Van den Brink, T.L. Estes, K. Gallagher, R. O'Connor, F. Verdonck, 2010. Bounding uncertainty analyses. In: Application of uncertainty analysis to ecological risks of pesticides / Warren-Hicks, W.J., A. Hart. Pensacola and Boca Raton, FL.: SETAC and CRC Press, 2010 -

Flage R., Aven T, Zio E., 2009. Alternative representations of uncertainty in reliability and risk analysis - review and discussion. In: Martorell S, Guedes Soares C, Barnett J, editors. Valencia, Spain, 22-25 September 2008 Safety, reliability and risk analysis. Theory, methods and applications. Proceedings of the European safety and reliability conference 2009 (ESREL 2009). London: CRC Press, p. 2081-91.

Flage, P. Baraldi, E. Zio, T. Aven, 2010. Possibility-probability transformation in comparing different approaches to the treatment of epistemic uncertainties in a fault tree analysis, in: B. Ale, I.A. Papazoglu, E. Zio (Eds.), Reliability, Risk and Safety - Proceedings of the European Safety and Reliability (ESREL) 2010 Conference, Rhodes, Greece, 5-9 September 2010, pp. 714-721, Taylor \& Francis Group, London, United Kingdom, 2010, ISBN 978-0-415-604277.

Flage, P. Baraldi, E. Zio, T. Aven, 2013. Probability and possibility-based representations of uncertainty in fault tree analysis. Risk Analysis, 33(1), pp. 121-33.

Gelman A., et al., 2004. Bayesian data analysis. Second Ed. Chapman \& Hall, CRC.

Gelman, A., 2006. Prior distributions for variance parameters in hierarchical models. Bayesian Analysis, 1(3), pp. 515-533.

Gelman, A., Meng, XL, Stern, H., 1996. Posterior predictive assessment of model fitness via realized discrepancies. Stat Sin: 733-806. 
Gelman, A., van Dyk, D., Huang, Z., Boscardin, W.J., 2008. Using Redundant Parameterizations to Fit Hierarchical Models. Journal of Computational and Graphical Statistics, Volume 17, Number 1, Pages 95-122.

Gill, J., 2002. Bayesian methods: a social and behavioral sciences approach, Chapman \& Hall/CRC, Boca Raton, FL.

Guyonnet, D., Bourgine, B., Dubois, D., Fargier, H., Côme, B., Chilès, J.P., 2003. Hybrid approach for addressing uncertainty in risk assessments. Journal of the Environmental Engineering Division, ASCE 129, pp. 68-78.

Helton, J.D. Johnson, W.L. Oberkampf, C.J. Sallaberry, 2008. Representation of Analysis Results Involving Aleatory and Epistemic Uncertainty, Sandia National Laboratories, Technical Report SAND2008-4379, Albuquerque, New Mexico.

Helton, J.D. Johnson, W.L. Oberkampf, C.B. Storlie, 2007. A sampling-based computational strategy for the representation of epistemic uncertainty in model predictions with evidence theory, Computer Methods in Applied Mechanics and Engineering, 196: 3980-98.

Hou, Y. and Yang, B., 2010. Probability-Possibility Transformation for Small Sample Size Data. 2010 Seventh International Conference on Fuzzy Systems and Knowledge Discovery (FSKD 2010), pp. 1720-1724.

Jalal-Kamali, A., and Kreinovich, V., 2013. Estimating correlation under interval uncertainty. Mechanical Systems and Signal Processing, 37(1-2), 43-53.

Jaworska, J.S., Aldenberg, T., 2000. Estimation of HC5 taking into account uncertainties of individual dose response curves and species sensitivity distribution. Presentation at the 2000 Society for Risk Analysis annual meeting, Arlingotn, Virginia, http://www.riskworld.com/Abstract/2000/SRAam00/ab0ac164.htm.

Johnson Valen, E., 2004. A Bayesian $\chi^{2}$ test for goodness-of-fit. Ann Stat, 32: pp. 2361-84.

Kalos, M.H., P.A. Whitlock, 1986. Monte Carlo methods. Volume I: Basics, Wiley, New York, NY. 
983 Kelly, D.L., Smith, C.L, 2009. Bayesian inference in probabilistic risk assessment — The current $984 \quad$ state of the art. Reliability Engineering and System Safety, 94, pp. 628-643.

985 Kelly, D.L., Smith, C.L, 2011. Bayesian Inference for Probabilistic Risk Assessment: A 986 Practitioner's Guidebook. Springer-Verlag: London, UK.

987 Kentel, E., M.M. Aral, 2004. Probabilistic-fuzzy health risk modeling. Stoch. Envir. Res. and Risk $988 \quad$ Ass., Vol. 18: pp. 324-338.

989 Kentel, E., M.M. Aral, 2005. 2D Monte Carlo versus 2D Fuzzy Monte Carlo Health Risk 990 Assessment, Internat. J. Stochastic Environ. Res. Risk Assess., 19, pp. 86-96.

991 Kentel, E., M.M. Aral, 2007. Risk tolerance measure for decision-making in fuzzy analysis: a health 992 risk assessment perspective. Stoch. Environ Res. Ris. Assess., Vol. 21: pp. 405-417.

993 Klir, B. Yuan, 1995. Fuzzy Sets and Fuzzy Logic: Theory and Applications, Prentice-Hall, Upper $994 \quad$ Saddle River, NJ.

995 Kozine, I., Filimonov, Y., 2000. Imprecise reliabilities: experiences and advances, Reliab. Eng. $996 \quad$ Syst. Saf., 67, 75-83.

997 Kozine, I. O., and Utkin, L. V., 2002. Processing unreliable judgements with an imprecise 998 hierarchical model. Risk, Decision and Policy, 7(03), 325-339.

999 Kuznetsov, V. P., 1991. Interval statistical models (in Russian), Radio i Svyaz, Moscow.

1000 Kwakernaak, H., 1978. Fuzzy Random Variables-I. Definitions and Theorems, Information 1001 Sciences 15, 1-29.

Lapointe S. and Bobeè B., 2000. Revision of possibility distributions: A Bayesian inference pattern. Fuzzy Sets and Systems, 116, pp. 119-140.

1004 Le Duy, T.D., Vasseur, D., Couplet, M., Dieulle, L., Bérenguer, C., 2011. A study on updating 1005 belief functions for pa-rameter uncertainty representation in Nuclear Probabilistic Risk Assessment. In F. Coolin, G. De Cooman, T. Fetz, M. Oberguggenberger (Eds.), Proceedings of the 7th Interna-tional Symposium on Imprecise Probability: Theories and Applications, 2528 July 2011, Innsbruck, Austria: 247-256. Innsbruck, Austria: SIPTA. 
1009 Limbourg, P., E. de Rocquigny, 2010. Uncertainty analysis using evidence theory - confronting

1010

1011

1012

1013

1014

1015

1016

1017

1018

1019

1020

1021

1022

1023

1024

1025

1026

1027

1028

1029

1030

1031

1032

1033

level-1 and level-2 approaches with data availability and computational constraints, Reliability Engineering and System Safety, Vol. 95, Issue 5, pp. 550-564.

Lindley, D.V., 2000. The philosophy of statistics. The Statistician, 49(3): 293-337.

Lindley, D. V., 2006. Understanding uncertainty, Wiley, Hoboken, NJ.

Masegosa, A., S. Moral, 2014. Imprecise probability models for learning multinomial distributions from data. Applications to learning credal networks. International Journal of Approximate Reasoning, Volume 55, Issue 7, Pages 1548-1569.

Masson, M.H. and Denoeux, T., 2006. Inferring a possibility distribution from empirical data. Fuzzy Sets and Systems, 157, pp. 319-340.

Mauris, G., 2008. Inferring a Possibility Distribution from Very Few Measurements. In: D. Dubois et al. (Eds.), Soft Methods for Hand. Var. and Imprecision, ASC 48, pp. 92-99, SpringerVerlag, Berlin, Heidelberg.

Mehl, C. H., 2013. P-boxes for cost uncertainty analysis. Mechanical Systems and Signal Processing, 37(1-2), 253-263.

Molchanov, I., 2005. Theory of Random Sets, Springer, New York.

Möller, B., M. Beer, 2004. Fuzzy Randomness: Uncertainty in Civil Engineering and Computational Mechanics, Springer, Berlin.

Möller, B., M. Beer, 2008. Engineering computation under uncertainty - Capabilities of nontraditional models. Computers and Structures, Vol. 86, pp. 1024-1041.

Moller, B., W. Graf, M. Beer, 2003. Safety assessment of structures in view of fuzzy randomness. Computers and Structures, Vol. 81, pp. 1567-1582.

Moller, B., M. Beer, W. Graf, J.U. Sickert, 2006. Time-dependent reliability of textile-strengthened RC structures under consideration of fuzzy randomness. Computers and Structures, Vol. 84, pp. 585-603. 
Moral, S., 2014. Comments on "Likelihood-based belief function: Justification and some extensions to low-quality data" by Thierry Denoeux. International Journal of Approximate Reasoning, Volume 55, Issue 7, Pages 1591-1593.

Muscolino, G., and Sofi, A., 2013. Bounds for the stationary stochastic response of truss structures with uncertain-but-bounded parameters. Mechanical Systems and Signal Processing, 37(1-2), 163-181.

NUREG-CR-6850, 2005. EPRI/NRC-RES Fire PRA methodology for nuclear power facilities, Volume 2: detailed methodology. US Nuclear Regulatory Commission.

NASA, 2010. Risk-Informed Decision Making Handbook. NASA/SP-2010-576 - Version 1.0, April 2010.

Pannier, S., Waurick, M., Graf, W., and Kaliske, M., 2013. Solutions to problems with imprecise data" - An engineering perspective to generalized uncertainty models. Mechanical Systems and Signal Processing, 37(1-2), 105-120.

Pasanisi, A., E. de Rocquigny, N. Bousquet, E. Parent, 2009. Some useful features of the Bayesian setting while dealing with uncertainties in industrial practice, Proceedings of the ESREL 2009 Conference, Prague, Czech Republic, 7-10 September 2009, pp. 1795-1802.

Pasanisi, A., Keller, M., Parent, M., 2012. Estimation of a quantity of interest inuncertainty analysis: Some help from Bayesian decision theory. Reliability Engineering and System Safety, 100, pp. 93-101.

Pedroni, N., Zio, E., 2012. Empirical comparison of methods for the hierarchical propagation of hybrid uncertainty in risk assessment, in presence of dependences. International Journal of Uncertainty, Fuzziness and Knowledge-Based Systems, Vol. 20, Issue 4, 2012, pp. 509-557.

Pedroni, N., E. Zio, E. Ferrario, A. Pasanisi, M. Couplet, 2013. Hierarchical propagation of probabilistic and non-probabilistic uncertainty in the parameters of a risk model. Computers and Structures, Volume 126, pp. 199-213. 
1059 Pedroni, N., E. Zio, A. Pasanisi, M. Couplet, 2014. Bayesian update of the parameters of probability 1060 distributions for risk assessment in a two-level hybrid probabilistic-possibilistic uncertainty 1061 framework. In: R.D.J.M. Steenbergen, P.H.A.J.M. van Gelder, S. Miraglia and A. C.W.M. $3295-3302$.

Puri, M.L., D.A. Ralescu, 1986. Fuzzy random variables, Journal of Mathematical Analysis and Applications 114, 409-422.

Quaeghebeur and de Cooman, 2005. Imprecise probability models for inference in exponential families. Electronic Proceedings of the 4th International Symposium on Imprecise Probabilities and Their Applications, Pittsburgh, Pennsylvania, 2005.

Ralescu, D., 2002. Average level of a fuzzy set, in: Statistical Modeling, Analysis and Management of Fuzzy Data, eds. C. Bertoluzza, M.A. Gil, D.A. Ralescu (Springer, Heidelberg) pp. 119_ 126.

Reid, S. G., 2013. Probabilistic confidence for decisions based on uncertain reliability estimates. Mechanical Systems and Signal Processing, 37(1-2), 229-239.

Sankararaman, S., and Mahadevan, S., 2013. Distribution type uncertainty due to sparse and imprecise data. Mechanical Systems and Signal Processing, 37(1-2), 182-198.

Sentz, S. Ferson, 2002. Combination of Evidence in Dempster-Shafer Theory, Sandia National Laboratories, Technical Report SAND 2002-0835, Albuquerque, New Mexico.

Serrurier, M., and Prade, H., 2011. Maximum-Likelihood Principle For Possibility Distributions Viewed As Families Of Probabilities. 2011 IEEE International Conference on Fuzzy Systems June 27-30, 2011, Taipei, Taiwan, pp. 2987-2993.

Shirley, K.E., Gelman, A., 2015. Hierarchical models for estimating state and demographic trends in US death penalty public opinion. J. R. Statist. Soc. A, 178(1), pp. 1-28. 
1085 Siu, N., Kelly, D., 1998. Bayesian parameter estimation in probabilistic risk assessment. Reliability Engineering and System Safety, 62, pp. 89-116.

1087 Smets, P., 1993. Belief Functions: The Disjunctive Rule of Combination and the Generalized Bayesian Theorem. International Journal of Approximate Reasoning, 9, pp. 1-35.

Stein M. \& M. Beer, 2011. Bayesian quantification of inconsistent information. Applications of Statistics and Probability in Civil Engineering - Faber, Köhler \& Nishijima (eds), Taylor \& Francis Group, London, ISBN 978-0-415-66986-3. Information Sciences: http://dx.doi.org/10.1016/j.ins.2013.02.024.

USNRC, 2002. An approach for using probabilistic risk assessment in risk-informed decisions on plant-specific changes to the licensing basis. NUREG-1.174 - Revision 1, US Nuclear Regulatory Commission, Washington, DC.

USNRC, 2009. Guidance on the Treatment of Uncertainties Associated with PRAs in RiskInformed Decision Making. NUREG-1855, US Nuclear Regulatory Commission, Washington, DC.

Viertl, R., 1996. Statistical Methods for Non-Precise Data. CRC Press, Boca Raton, New York, London, Tokyo.

Viertl, R., 1997. On statistical inference for non precise data. Environmetrics, 8, pp. 541-568.

Viertl, R., 1999. Statistics and integration of fuzzy functions. Environmetrics, 10, pp. 487-491.

Viertl, R., 2008a. Foundations of Fuzzy Bayesian Inference. Journal of Uncertain Systems, 2(3), pp. Variability and Imprecision, ASC 48, pp. 10-15.

Viertl, R., 2011. Statistical Methods for Fuzzy Data, Wiley, Chichester.

1109 Viertl, R. \& Hareter, D., 2004a. Fuzzy information and imprecise probability. ZAMM, Z. Angew. 
1111 Viertl, R. \& Hareter, D., 2004b. Generalized Bayes-theorem for non-precise a-priori distribution, $1112 \quad$ Metrika, 59(3), pp. 263-273.

1113 Viertl R. and H. Hule, 1991. On Bayes’ theorem for fuzzy data. Stat. Pap. 32.

1114 Walley, P., 1991. Statistical reasoning with imprecise probabilities, Chapman and Hall, New York. 1115 Walley, P., 1996. Inferences from multinomial data: learning about a bag of marbles (with 1116 discussion), J. R. Stat. Soc. B 58, 3-57.

1117 Walter, G., and Augustin, T., 2009. Imprecision and Prior-data Conflict in Generalized Bayesian 1118 Inference. Journal of Statistical Theory and Practice, Volume 3, No.1, pp. 255-271.

1119 Weichselberger, K., 2000. The theory of interval-probability as a unifying concept for uncertainty, 1120 Int. J. Approx. Reasoning, 24(2-3), 149-170.

1121 Zhang, H., Dai, H., Beer, M., and Wang, W., 2013. Structural reliability analysis on the basis of 1122 small samples: An interval quasi-Monte Carlo method. Mechanical Systems and Signal $1123 \quad$ Processing, 37(1-2), 137-151.

1124

1125 


\section{Figure captions list}

1129 Fig. 1. Top left: triangular possibility distribution $\pi^{\gamma}(\gamma)$ of the epistemically-uncertain parameter $\gamma$ of the Gumbel probability distribution of $Y$; in evidence the $\alpha$-cuts of level $\alpha=0,0.5$ and 1 . Top right: four PDFs belonging to the family $\left\{p^{Y}(y \mid \gamma, \delta)\right\}_{\alpha=0}$ and four PDFs belonging to the family $\left\{p^{Y}(y \mid \gamma, \delta)\right\}_{\alpha=0.5}$. Bottom: bounding upper and lower CDFs of $Y, \bar{F}_{\alpha}^{Y}(y)=P l_{\alpha}^{Y}(Z)$ and $\underline{F}_{\alpha}^{Y}(y)=\operatorname{Bel}_{\alpha}^{Y}(Z), Z=(-\infty, y]$, built in correspondence of the $\alpha$-cuts of level $\alpha=0,0.5$ and 1 of $\pi^{\gamma}(\gamma)$; the plausibility and belief functions $\mathrm{Pl}^{Y}(Z)$ and $\mathrm{Bel}^{Y}(Z), Z=(-\infty, y]$, are also shown

Fig. 2. Identification of the P-dimensional $\alpha$-cut $A_{\alpha}^{\theta \mid y}$ of the posterior possibility distribution of $\boldsymbol{\theta}$ as the hull enveloping $T=20$ point estimates $\boldsymbol{\theta}_{l, \alpha} \mid \boldsymbol{y}, l=1,2, \ldots, 20$ (dots), generated by the repeated application of the Bayes' theorem in the hybrid probabilistic-possibilistic approach $(B)$, in the particular case of $P=2$ parameters $\theta_{1}$ and $\theta_{2}$. The corresponding $\alpha$-cut $A_{\alpha, \text { Cart }}^{\theta_{1}, \theta_{2} \mid y}$ generated by the Cartesian product of the (one-dimensional) $\alpha$-cuts $A_{\alpha}^{\theta_{1} \mid y}$ and $A_{\alpha}^{\theta_{2} \mid y}$ of the marginal possibility distributions is also shown

Fig. 3. Prior and posterior possibility distributions of the epistemically-uncertain parameters of the aleatory PDFs of $Y_{1}=Q$ (top row, left and right column), $Y_{2}=Z_{m}$ (middle-top row, left and right column $), Y_{3}=Z_{v}$ (middle-bottom row, left and right column) and $Y_{4}=K_{s}$ (bottom row, left and right column). The point estimates of the parameters obtained by the classical MLE method are also shown for comparison. Adapted and extended from [Pedroni et al., 2014] 
1150 Fig. 4. Exemplary $\alpha$-cuts of level $\alpha=0.05$, 0.50 and 0.95 of the joint posterior possibility distributions of the parameters of the PDFs of $Y_{1}=Q$ (top left), $Y_{2}=Z_{m}$ (top right), $Y_{3}=Z_{v}$ (bottom left) and $Y_{4}=K_{S}($ bottom right), produced by Approaches $A$ (solid lines) and $B$ (dashed lines)

1155 Fig. 5. Upper and lower CDFs, $\bar{F}^{Y_{j}}\left(y_{j}\right)$ and $\underline{F}^{Y_{j}}\left(y_{j}\right), j=1,2,3$, 4, of the uncertain input variables $Y_{1}=Q($ top left $), Y_{2}=Z_{m}($ top right $), Y_{3}=Z_{v}($ bottom left $)$ and $Y_{4}=K_{s}($ bottom right $)$ before and after the Bayesian update performed by Approaches $A$ and $B$

1159 Fig. 6. Plausibility and belief functions, $P l^{Z_{c}}\left(\left(-\infty, z_{c}\right]\right)$ and $\mathrm{Bel}^{Z_{c}}\left(\left(-\infty, z_{c}\right]\right)$, of the maximal water $1160 \quad$ level of the river $Z_{c}$ before and after the Bayesian update performed by Approaches $A$ and $B$. $1161 \quad$ Adapted and extended from [Pedroni et al., 2014]

1162 

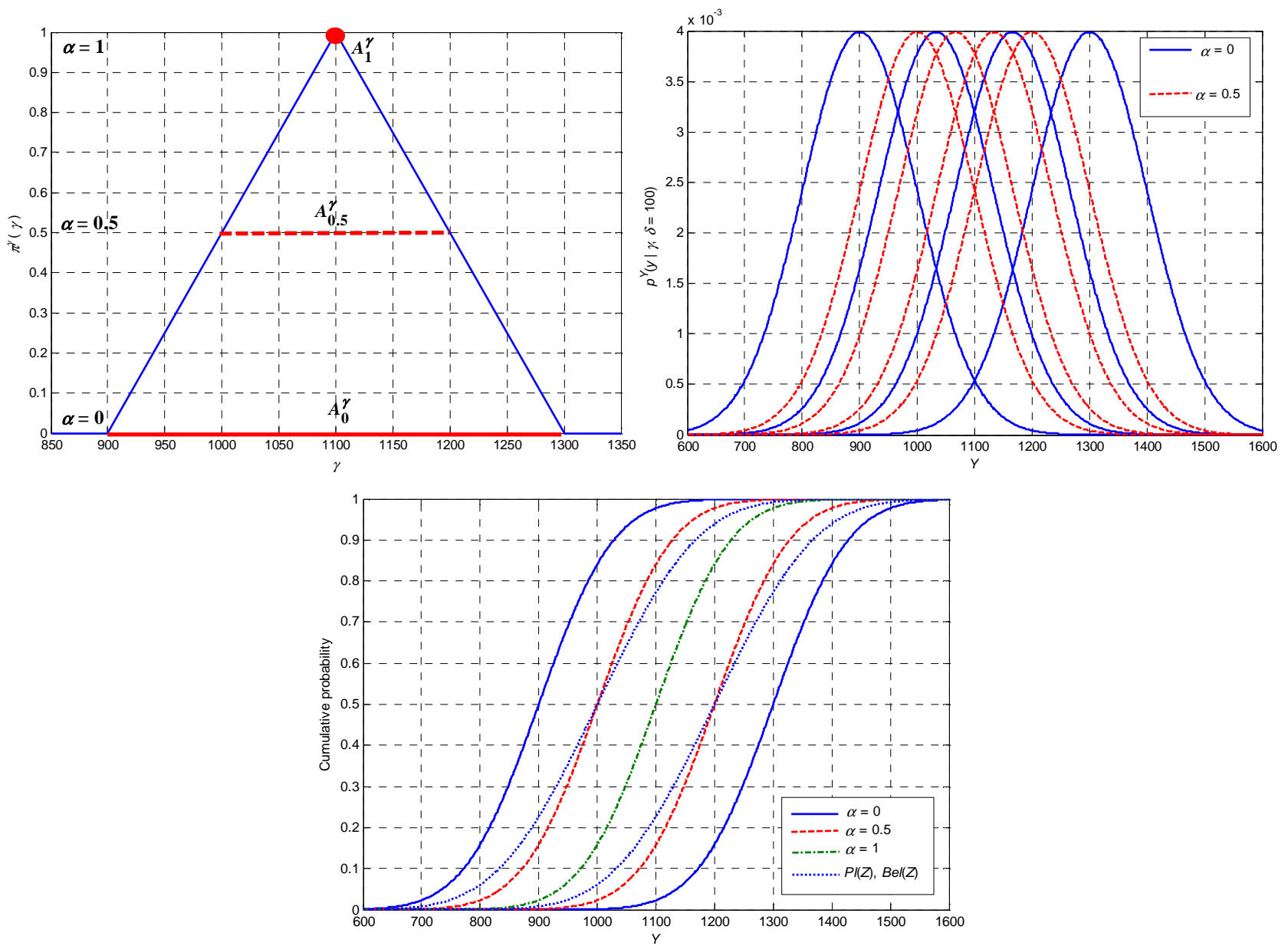

1168 Fig. 1. Top left: triangular possibility distribution $\pi^{\gamma}(\gamma)$ of the epistemically-uncertain parameter $\gamma$ of the Gumbel probability distribution of $Y$; in evidence the $\alpha$-cuts of level $\alpha=0,0.5$ and 1 . Top right: four PDFs belonging to the family $\left\{p^{Y}(y \mid \gamma, \delta)\right\}_{\alpha=0}$ and four PDFs belonging to the family $\left\{p^{Y}(y \mid \gamma, \delta)\right\}_{\alpha=0.5}$. Bottom: bounding upper and lower $C D F$ s of $Y, \bar{F}_{\alpha}^{Y}(y)=P l_{\alpha}^{Y}(Z)$ and of $\pi^{\gamma}(\gamma)$; the plausibility and belief functions $\mathrm{Pl}^{Y}(Z)$ and $\mathrm{Bel}^{Y}(Z), Z=(-\infty, y]$, are also shown 


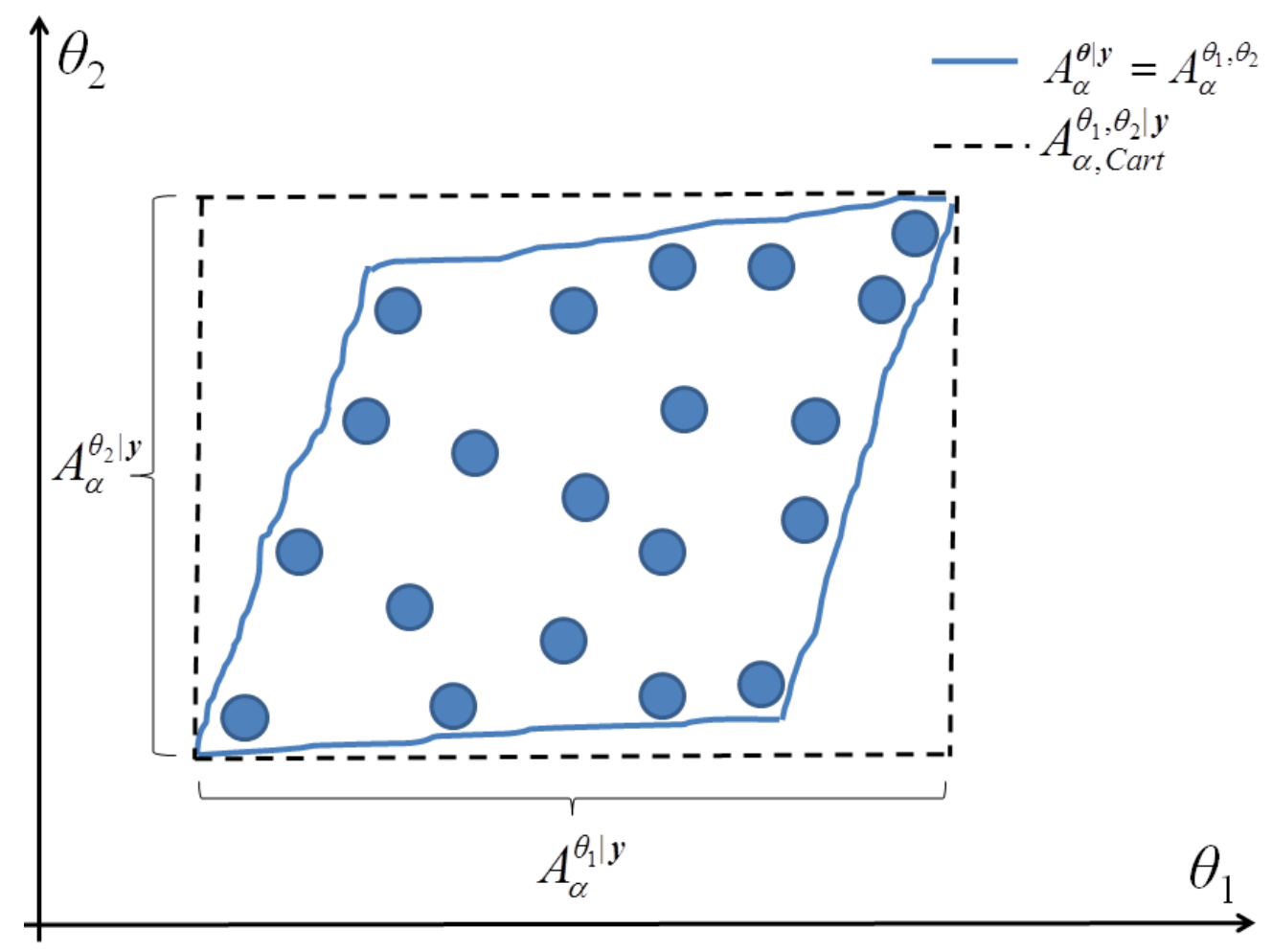

1177 Fig. 2. Identification of the P-dimensional $\alpha$-cut $A_{\alpha}^{\theta \mid y}$ of the posterior possibility distribution of $\boldsymbol{\theta}$ as 1178 the hull enveloping $T=20$ point estimates $\boldsymbol{\theta}_{l, \alpha} \mid \boldsymbol{y}, l=1,2, \ldots, 20$ (dots), generated by the 1179 repeated application of the Bayes' theorem in the hybrid probabilistic-possibilistic approach $1180(B)$, in the particular case of $P=2$ parameters $\theta_{1}$ and $\theta_{2}$. The corresponding $\alpha$-cut $A_{\alpha, \text { Cart }}^{\theta_{1}, \theta_{2} \mid y}$ 1181 generated by the Cartesian product of the (one-dimensional) $\alpha$-cuts $A_{\alpha}^{\theta_{1} \mid y}$ and $A_{\alpha}^{\theta_{2} \mid y}$ of the 1182 marginal possibility distributions is also shown 1183 

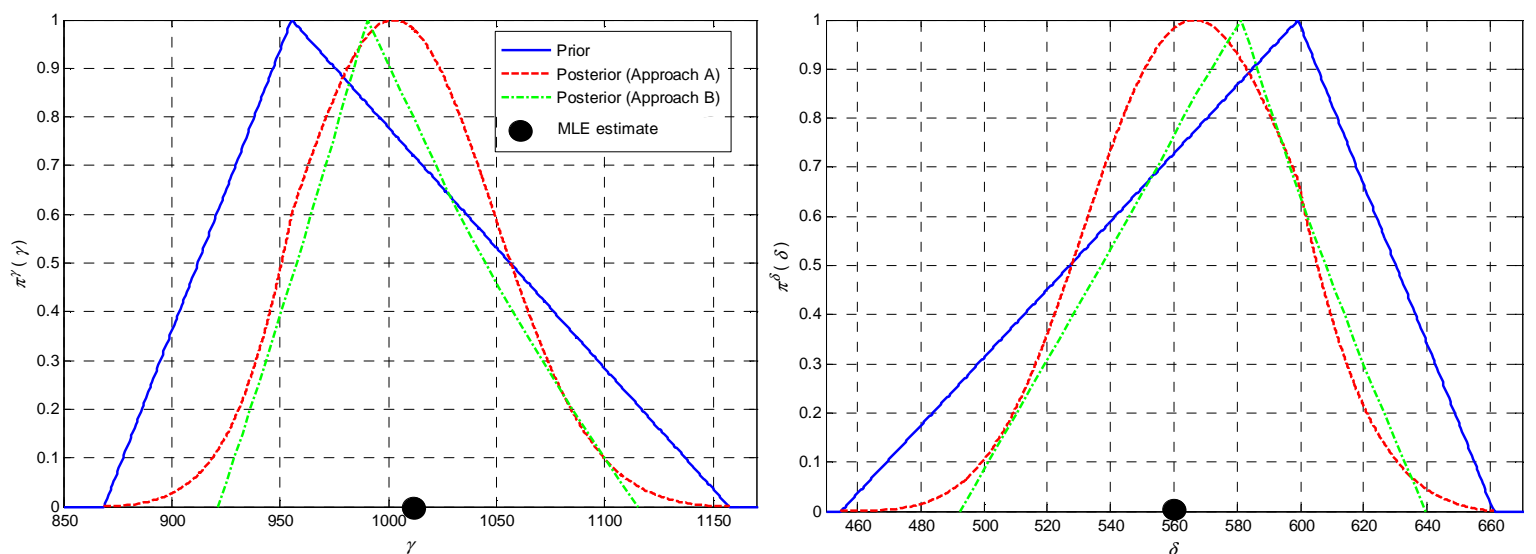

1185
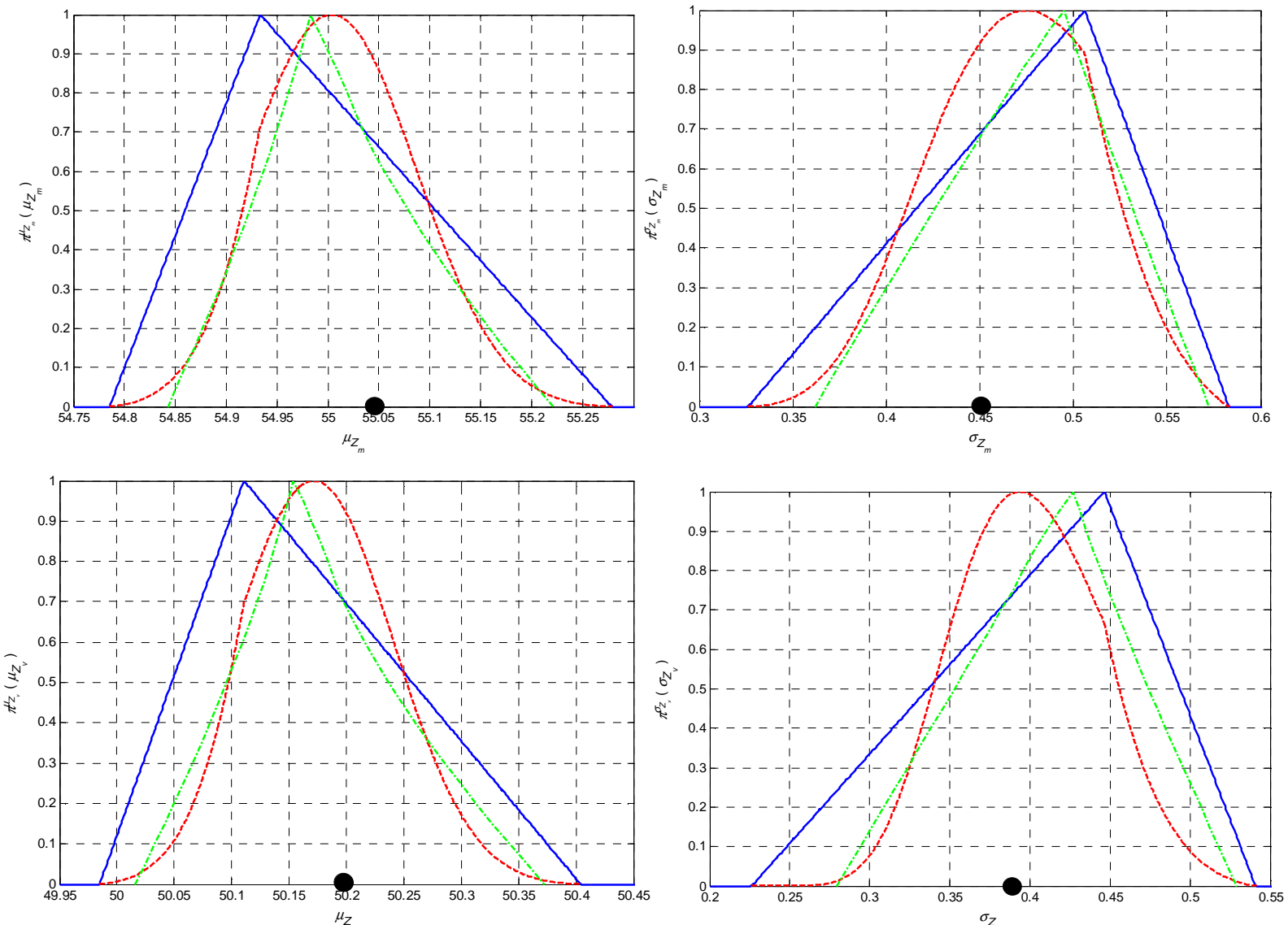

1186
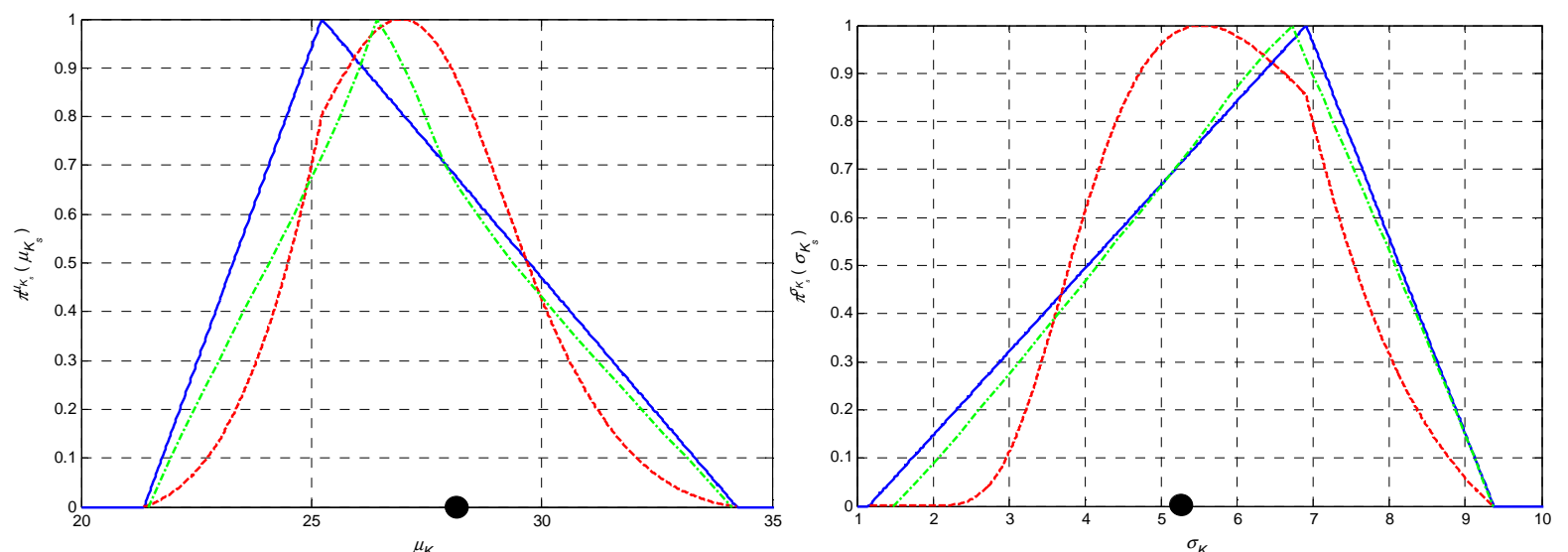
1189 Fig. 3. Prior and posterior possibility distributions of the epistemically-uncertain parameters of the $1190 \quad$ aleatory PDFs of $Y_{1}=Q$ (top row, left and right column), $Y_{2}=Z_{m}$ (middle-top row, left and 1191 right column), $Y_{3}=Z_{v}$ (middle-bottom row, left and right column) and $Y_{4}=K_{s}$ (bottom row, 1192 left and right column). The point estimates of the parameters obtained by the classical MLE 1193 method are also shown for comparison. Adapted and extended from [Pedroni et al., 2014] 1194 
1195

1196
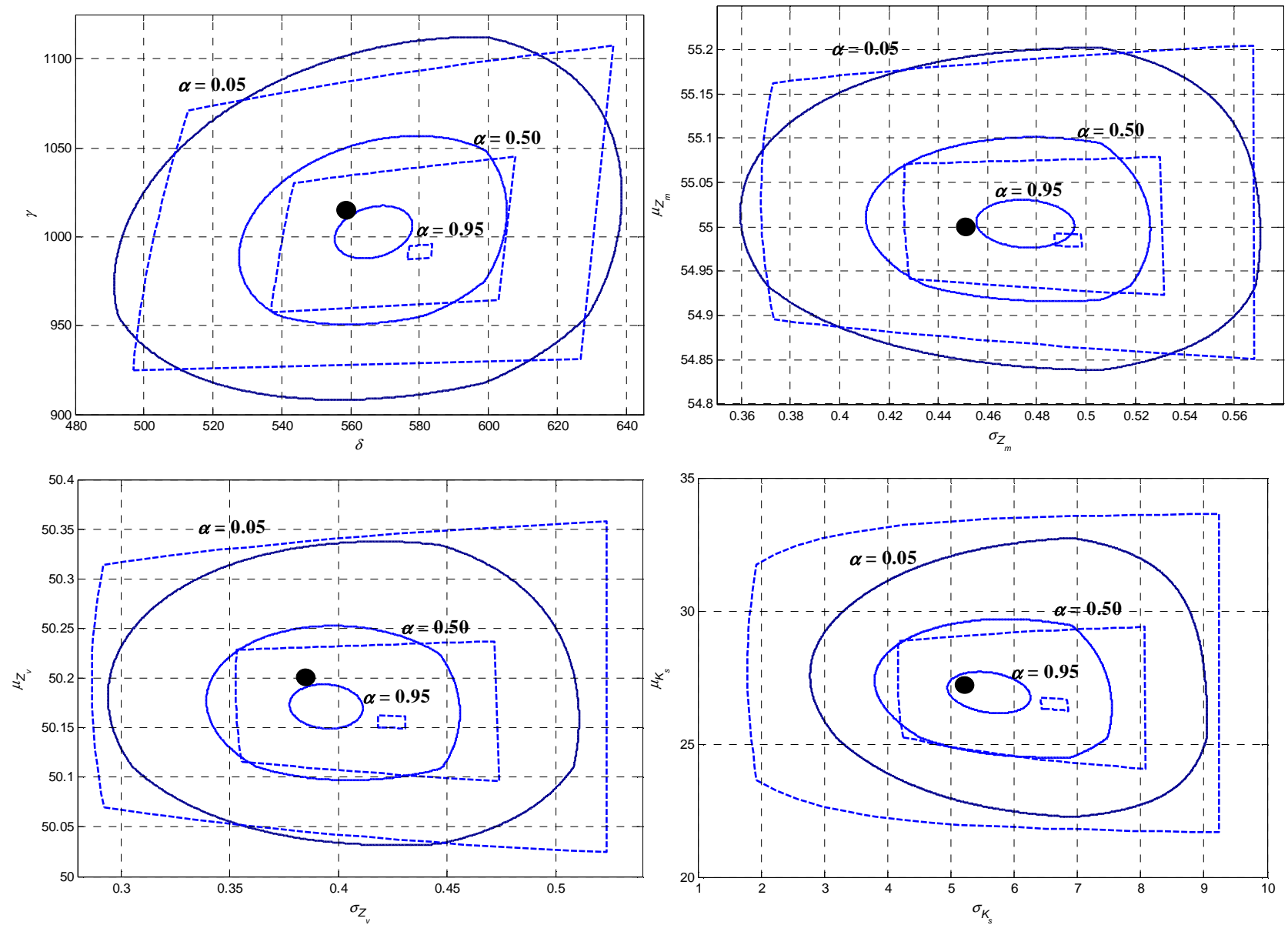

1198 Fig. 4. Exemplary $\alpha$-cuts of level $\alpha=0.05,0.50$ and 0.95 of the joint posterior possibility distributions of the parameters of the PDFs of $Y_{1}=Q$ (top left), $Y_{2}=Z_{m}$ (top right), $Y_{3}=Z_{v}$ (bottom left) and $Y_{4}=K_{S}($ bottom right), produced by Approaches $A$ (solid lines) and $B$ (dashed lines) 
1203

1204
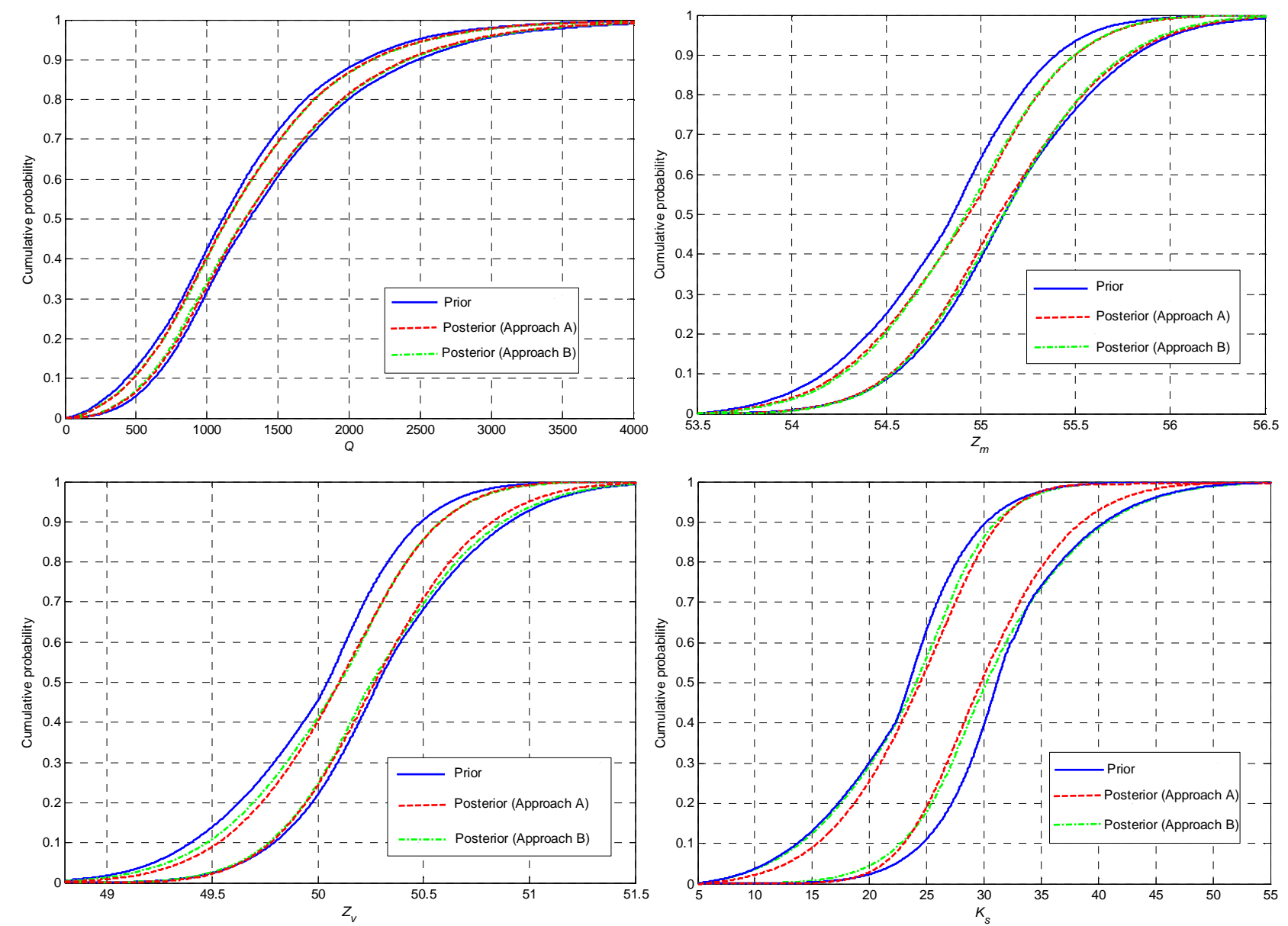

1206 Fig. 5. Upper and lower CDFs, $\bar{F}^{Y_{j}}\left(y_{j}\right)$ and $\underline{F}^{Y_{j}}\left(y_{j}\right), j=1,2,3$, 4, of the uncertain input variables $Y_{1}=Q($ top left $), Y_{2}=Z_{m}($ top right $), Y_{3}=Z_{v}($ bottom left $)$ and $Y_{4}=K_{s}($ bottom right $)$ before and after the Bayesian update performed by Approaches A and B 


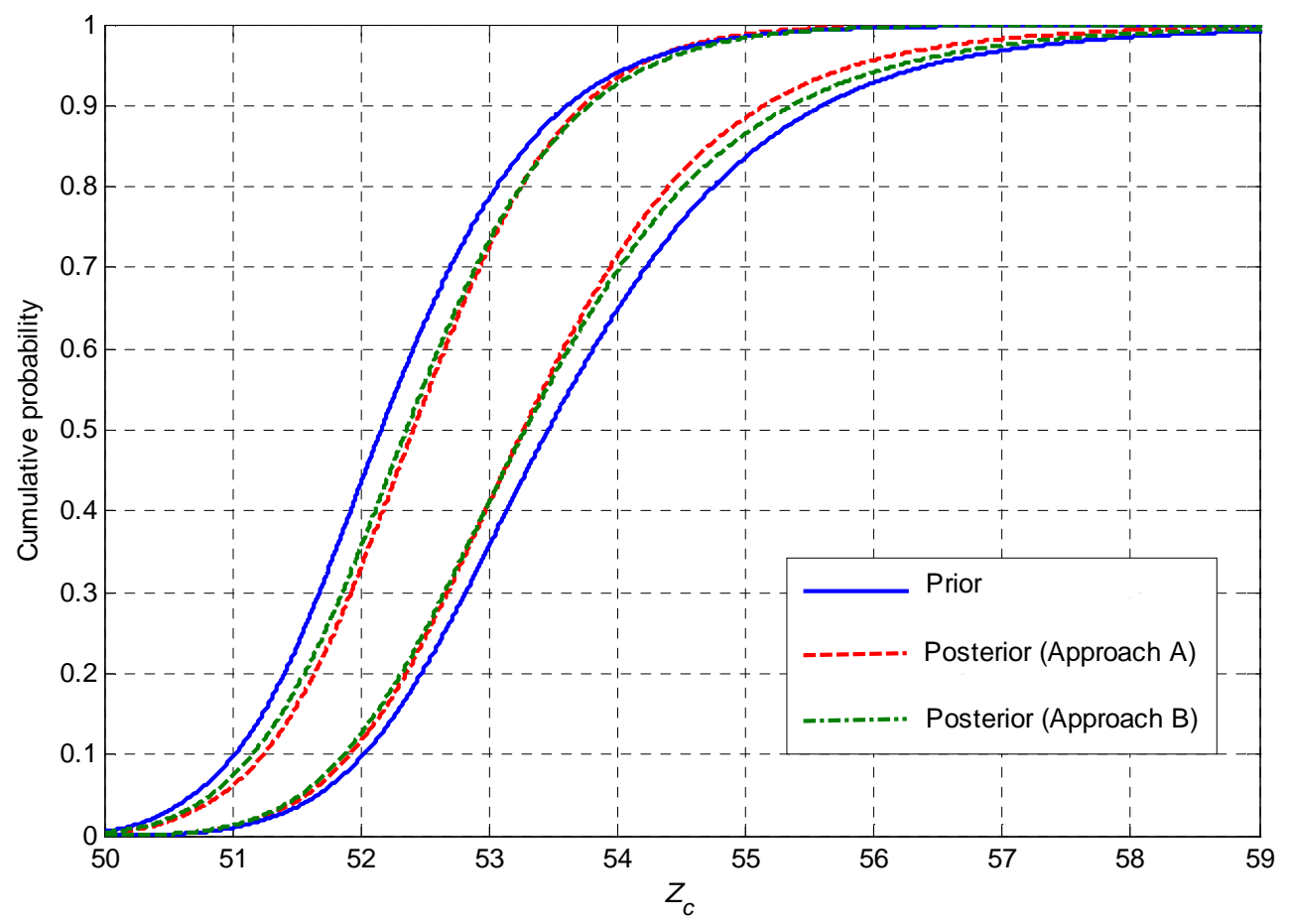

1211 Fig. 6. Plausibility and belief functions, $\operatorname{Pl}^{Z_{c}}\left(\left(-\infty, z_{c}\right]\right)$ and $\operatorname{Bel}^{Z_{c}}\left(\left(-\infty, z_{c}\right]\right)$, of the maximal water $1212 \quad$ level of the river $Z_{c}$ before and after the Bayesian update performed by Approaches $A$ and $B$. $1213 \quad$ Adapted and extended from [Pedroni et al., 2014] 


\section{Purely possibilistic method (A)}

\begin{tabular}{|c|c|}
\hline Prior & Possibility distribution \\
\hline Likelihood & $\begin{array}{l}\text { Possibility distribution: } \\
\text { - Probability-possibility transformation in this } \\
\text { paper (e.g., normalization: see also [Denoeux, 2014]) }\end{array}$ \\
\hline
\end{tabular}

Posterior Possibility distribution
Hybrid probabilistic-possibilistic method (B)

Fuzzy Probability Distribution Function

Probabilistic function

Fuzzy Probability Distribution Function: - Expected value: possibility distribution

\section{Table 1. Characteristics of the purely possibilistic (A) and the hybrid probabilistic and possibilistic}

(B) approaches 


\begin{tabular}{|c|c|c|}
\hline & & Purely possibilistic method (Approach A) \\
\hline & Aleatory uncertainty & Epistemic uncertainty (Purely possibilistic priors) \\
\hline \multirow{2}{*}{$Q$} & \multirow{2}{*}{$\operatorname{Gum}(\gamma, \delta)$} & $\pi^{\gamma}(\gamma)=T R\left(a_{\gamma}, c_{\gamma}, b_{\gamma}\right)=T R(869,955,1157)$ \\
\hline & & $\pi^{\delta}(\delta)=T R\left(a_{\delta}, c_{\delta}, b_{\delta}\right)=T R(455,600,660)$ \\
\hline \multirow{2}{*}{$Z_{m}$} & \multirow{2}{*}{$N\left(\mu_{\mathrm{Zm}}, \sigma_{\mathrm{Zm}}\right)$} & $\pi^{\mu_{z n}}\left(\mu_{Z m}\right)=T R\left(a_{\mu_{z n}}, c_{\mu_{z n}}, b_{\mu_{z n}}\right)=T R(54.78,54.93,55.28)$ \\
\hline & & $\pi^{\sigma_{z n}}\left(\sigma_{Z m}\right)=T R\left(a_{\sigma_{Z n}}, c_{\sigma_{z n}}, b_{\sigma_{Z n}}\right)=T R(0.33,0.51,0.58)$ \\
\hline \multirow{2}{*}{$Z_{v}$} & \multirow{2}{*}{$N\left(\mu_{Z v}, \sigma_{Z v}\right)$} & $\pi^{\mu_{z_{v}}}\left(\mu_{\mathrm{zv}}\right)=\operatorname{TR}\left(a_{\mu_{\mathrm{zu}}}, c_{\mu_{\mathrm{zv}}}, b_{\mu_{\mathrm{zv}}}\right)=\operatorname{TR}(49.98,50.11,50.40)$ \\
\hline & & $\pi^{\sigma_{\mathrm{zv}}}\left(\sigma_{\mathrm{Zv}}\right)=\operatorname{TR}\left(a_{\sigma_{\mathrm{Zu}}}, c_{\sigma_{\mathrm{Zv}}}, b_{\sigma_{\mathrm{zv}}}\right)=\operatorname{TR}(0.23,0.45,0.54)$ \\
\hline \multirow{2}{*}{$\boldsymbol{K}_{s}$} & \multirow{2}{*}{$N\left(\mu_{K s}, \sigma_{K s}\right)$} & $\pi^{K_{s}}\left(\mu_{K_{s}}\right)=T R\left(a_{\mu_{k s}}, c_{\mu_{k s}}, b_{\mu_{k s}}\right)=T R(21.37,25.23,34.23)$ \\
\hline & & $\pi^{\sigma_{K s}}\left(\sigma_{K s}\right)=T R\left(a_{\sigma_{K s}}, c_{\sigma_{K s}}, b_{\sigma_{K s}}\right)=\operatorname{TR}(1.16,6.91,9.37)$ \\
\hline
\end{tabular}

\begin{tabular}{|c|c|c|c|}
\hline & \multirow{3}{*}{ Aleatory uncertainty } & \multicolumn{2}{|c|}{ Hybrid probabilistic-possibilistic method (Approach B) } \\
\hline & & \multicolumn{2}{|c|}{ Epistemic uncertainty (Fuzzy Probability Density Functions - FPDFs) } \\
\hline & & Probabilistic part & $\begin{array}{l}\text { Possibilistic part } \\
\end{array}$ \\
\hline \multirow{4}{*}{$Q$} & \multirow{4}{*}{$\operatorname{Gum}(\gamma, \delta)$} & \multirow{2}{*}{$p^{\gamma}(\gamma)=N\left(\mu_{\gamma}, \sigma_{\gamma}\right)$} & $\pi^{\mu_{\gamma}}\left(\mu_{\gamma}\right)=T R\left(a_{\mu_{\gamma}}, c_{\mu_{\gamma}}, b_{\mu_{\gamma}}\right)=T R(869,955,1157)$ \\
\hline & & & $\pi^{\sigma_{\gamma}}\left(\sigma_{\gamma}\right)=T R\left(a_{\sigma_{\gamma}}, c_{\sigma_{\gamma}}, b_{\sigma_{\gamma}}\right)=T R(39,52,57)$ \\
\hline & & \multirow{2}{*}{$p^{\delta}(\delta)=N\left(\mu_{\delta}, \sigma_{\delta}\right)$} & $\pi^{\mu_{s}}\left(\mu_{\delta}\right)=T R\left(a_{\mu_{s}}, c_{\mu_{s}}, b_{\mu_{s}}\right)=T R(455,600,660)$ \\
\hline & & & $\pi^{\sigma_{s}}\left(\sigma_{\delta}\right)=T R\left(a_{\sigma_{s}}, c_{\sigma_{s}}, b_{\sigma_{s}}\right)=T R(25,31,43)$ \\
\hline \multirow{4}{*}{$Z_{m}$} & \multirow{4}{*}{$N\left(\mu_{Z m}, \sigma_{Z m}\right)$} & \multirow{2}{*}{$p^{\mu_{2 m}}\left(\mu_{z m}\right)=N\left(\mu_{\mu_{2 m}}, \sigma_{\mu_{2 n}}\right)$} & 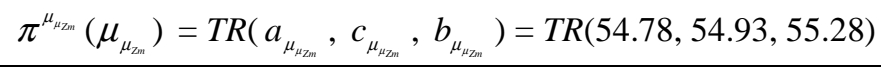 \\
\hline & & & 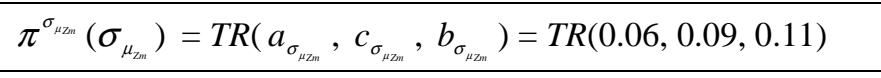 \\
\hline & & \multirow{2}{*}{$p^{\sigma_{2 n}}\left(\sigma_{Z m}\right)=N\left(\mu_{\sigma_{z m}}, \sigma_{\sigma_{z m}}\right)$} & 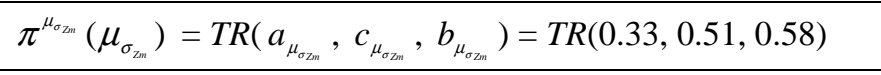 \\
\hline & & & $\pi^{\sigma_{\text {zzm }}}\left(\sigma_{\sigma_{\text {znn }}}\right)=T R\left(a_{\sigma_{\sigma_{z n}}}, c_{\sigma_{\sigma_{\text {zzn }}}}, b_{\sigma_{\sigma_{\text {zan }}}}\right)=T R(0.03,0.04,0.06)$ \\
\hline \multirow{4}{*}{$Z_{v}$} & \multirow{4}{*}{$N\left(\mu_{z v}, \sigma_{z v}\right)$} & \multirow{2}{*}{$p^{\mu_{\mu_{v}}}\left(\mu_{z_{v}}\right)=N\left(\mu_{\mu_{\mu_{v}}}, \sigma_{\mu_{\mu_{v}}}\right)$} & 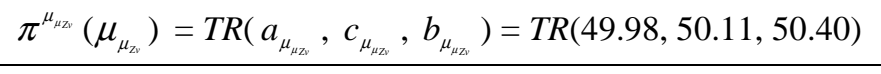 \\
\hline & & & 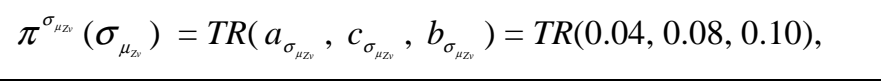 \\
\hline & & \multirow{2}{*}{$p^{\sigma_{\mathrm{zu}_{v}}}\left(\sigma_{z_{v}}\right)=N\left(\mu_{\sigma_{\mathrm{z}}}, \sigma_{\sigma_{\mathrm{zv}}}\right)$} & 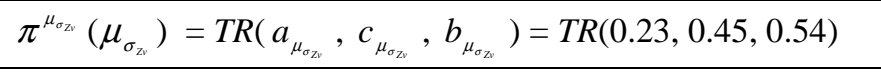 \\
\hline & & & 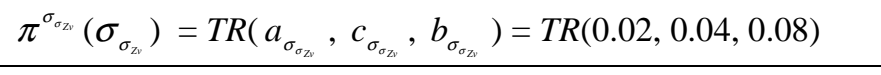 \\
\hline \multirow{4}{*}{$\boldsymbol{K}_{s}$} & \multirow{4}{*}{$N\left(\mu_{K s}, \sigma_{K s}\right)$} & \multirow{2}{*}{$p^{\mu_{k s}}\left(\mu_{K s}\right)=N\left(\mu_{\mu_{k s}}, \sigma_{\mu_{k s}}\right)$} & $\pi^{\mu_{\mu k E}}\left(\mu_{\mu_{k s}}\right)=T R\left(a_{\mu_{\mu k s}}, c_{\mu_{\mu k s}}, b_{\mu_{\mu k S}}\right)=T R(21.37,25.23,34.23)$ \\
\hline & & & $\pi^{\sigma_{\mu K \varepsilon}}\left(\sigma_{\mu_{k s}}\right)=T R\left(a_{\sigma_{\mu K s}}, c_{\sigma_{\mu k s}}, b_{\sigma_{\mu k s}}\right)=T R(0.50,2.80,3.79)$ \\
\hline & & \multirow{2}{*}{$p^{\sigma_{K s}}\left(\sigma_{K s}\right)=N\left(\mu_{\sigma_{K s}}, \sigma_{\sigma_{k s}}\right)$} & 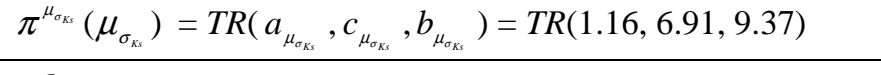 \\
\hline & & & $\pi^{\sigma_{\sigma_{K S}}}\left(\sigma_{\sigma_{K_{k s}}}\right)=T R\left(a_{\sigma_{\sigma_{K S}}}, c_{\sigma_{\sigma_{K S}}}, b_{\sigma_{\sigma_{K S}}}\right)=T R(0.10,0.86,2.64)$ \\
\hline
\end{tabular}

Table 2. Characteristics and parameters of the prior distributions of the uncertain variables $Y_{1}=$ 


\begin{tabular}{|c|c|c|c|c|c|c|c|c|}
\hline \multirow[b]{3}{*}{ Variable } & \multirow[b]{3}{*}{$\theta$} & \multirow[b]{3}{*}{ MLE $\hat{\theta}^{M L E}$} & \multicolumn{6}{|c|}{ Marginal possibility distributions update } \\
\hline & & & \multicolumn{3}{|c|}{ Most likely value, $c_{\theta}\left(d_{\theta}^{M L E}\right)$} & \multicolumn{3}{|c|}{ Area, $S_{\theta}\left(R_{\theta}\right)$} \\
\hline & & & Prior & Posterior A & Posterior B & Prior & Posterior A & Posterior B \\
\hline \multirow{2}{*}{$Q\left[\mathrm{~m}^{3} / \mathrm{s}\right]$} & $\gamma$ & 1013.21 & 955.55 & $1002.70(0.010)$ & $990.42(0.023)$ & 144.65 & $100.55(30.49)$ & $96.90(33.01)$ \\
\hline & $\delta$ & 558.48 & 599.15 & $566.35(0.014)$ & $581.40(0.041)$ & 103.35 & $76.94(25.56)$ & $73.65(28.74)$ \\
\hline \multirow{2}{*}{$Z_{m}[\mathrm{~m}]$} & $\mu_{Z m}$ & 55.03 & 54.93 & $55.00(5.45 \mathrm{e}-4)$ & $54.98(9.09 \mathrm{e}-4)$ & 0.25 & $0.190(24.00)$ & $0.195(22.00)$ \\
\hline & $\sigma_{Z m}$ & 0.45 & 0.51 & $0.47(0.044)$ & $0.50(0.111)$ & 0.12 & $0.110(8.33)$ & $0.115(4.17)$ \\
\hline \multirow{2}{*}{$Z_{v}[\mathrm{~m}]$} & $\mu_{Z v}$ & 50.19 & 50.11 & $50.17(3.98 \mathrm{e}-4)$ & $50.15(7.97 \mathrm{e}-4)$ & 0.21 & $0.165(21.43)$ & $0.170(19.05)$ \\
\hline & $\sigma_{Z v}$ & 0.38 & 0.45 & $0.39(0.026)$ & $0.43(0.132)$ & 0.16 & $0.121(24.38)$ & $0.125(21.88)$ \\
\hline \multirow{2}{*}{$K_{s}[/]$} & $\mu_{K s}$ & 27.80 & 25.24 & $26.95(0.031)$ & $26.43(0.049)$ & 6.45 & $5.40(16.28)$ & $5.80(10.08)$ \\
\hline & $\sigma_{K s}$ & 5.26 & 6.89 & $5.54(0.053)$ & $6.72(0.278)$ & 4.11 & $3.75(8.76)$ & $3.95(3.89)$ \\
\hline
\end{tabular}

1226 Table 3. Most likely values $c_{\theta}$ of the parameters $\theta=\gamma, \delta, \mu_{Z m}, \sigma_{Z m}, \mu_{Z v}, \sigma_{Z v}, \mu_{K_{s}}$ and $\sigma_{K s}$ of the aleatory PDFs of the input variables $Y_{1}=Q, Y_{2}=Z_{m}, Y_{3}=Z_{v}$ and $Y_{4}=K_{s}$ and areas $S_{\theta}$ underlying the corresponding (marginal) possibility distributions before and after the Bayesian update performed by Approaches A and B. The point estimates $\hat{\theta}^{M L E}$ obtained by the classical MLE method are also shown for comparison together with the values of the quantitative indicators $d_{\theta}^{M L E}(5)$ and $R_{\theta}(6)$ (in parentheses). Adapted and extended from 


\begin{tabular}{|c|c|c|c|c|}
\hline \multicolumn{5}{|c|}{ INPUT VARIABLES } \\
\hline Variable & Indicator & Prior & $\begin{array}{c}\text { Purely possibilistic approach (A) } \\
\text { (\% width reduction) }\end{array}$ & $\begin{array}{l}\text { Hybrid approach }(\mathrm{B}) \\
\text { (\% width reduction) }\end{array}$ \\
\hline \multirow{3}{*}{$Y_{1}=Q$} & $Q^{0.05}$ & {$[284.8,480.9]$} & {$[329.3,446.3](0.4033)$} & {$[322.9,434.1](0.4329)$} \\
\hline & $Q^{0.50}$ & {$[1110.5,1295.5]$} & {$[1143.6,1269.1](0.3216)$} & {$[1145.5,1263.7](0.3610)$} \\
\hline & $Q^{0.95}$ & {$[2478.2,2917.3]$} & {$[2553.4,2857.9](0.3065)$} & {$[2571.1,2879.0](0.2987)$} \\
\hline \multirow{3}{*}{$Y_{2}=Z_{m}$} & $Z_{m}{ }^{0.05}$ & {$[53.98,54.36]$} & {$[54.09,54.37](0.2631)$} & {$[54.06,54.36](0.2105)$} \\
\hline & $Z_{m}{ }^{0.50}$ & {$[54.85,55.12]$} & {$[54.92,55.09](0.4074)$} & {$[54.93,55.12](0.2592)$} \\
\hline & $Z_{m}{ }^{0.95}$ & {$[55.56,56.02]$} & {$[55.66,55.96](0.3478)$} & {$[55.67,55.99](0.3043)$} \\
\hline \multirow{3}{*}{$Y_{3}=Z_{v}$} & $Z_{v}^{0.05}$ & {$[49.22,49.64]$} & {$[49.35,49.64](0.3095)$} & {$[49.29,49.62](0.2142)$} \\
\hline & $Z_{v}^{0.50}$ & {$[50.04,50.29]$} & {$[50.10,50.25](0.4000)$} & {$[50.10,50.27](0.3199)$} \\
\hline & $Z_{v}^{0.95}$ & {$[50.63,51.09]$} & {$[50.72,51.00](0.3913)$} & {$[50.72,51.06](0.2608)$} \\
\hline \multirow{3}{*}{$Y_{4}=K_{s}$} & $K_{s}^{0.05}$ & {$[10.95,22.33]$} & {$[12.75,21.25](0.2530)$} & {$[11.15,20.38](0.1889)$} \\
\hline & $K_{s}^{0.50}$ & {$[23.49,31.15]$} & {$[24.43,29.73](0.3080)$} & {$[24.05,30.22](0.1945)$} \\
\hline & $K_{s}^{0.95}$ & {$[32.66,44.14]$} & {$[33.01,41.40](0.2691)$} & {$[33.32,44.14](0.0574)$} \\
\hline
\end{tabular}

Table 4. Intervals $\left[\left[\bar{F}^{Y_{j}}\right]^{-1}(\beta),\left[\underline{F}^{Y_{j}}\right]^{-1}(\beta)\right]$ for the $\beta \cdot 100$-th percentiles $Y_{j}^{\beta}, \beta=0.05,0.50$ and 0.95 , Approaches $A$ and $B$. The percentage reduction in the width of the intervals produced by 
OUTPUT VARIABLE $Z_{c}$

\begin{tabular}{ccccc}
\hline Variable & Indicator & Prior & $\begin{array}{c}\text { Purely possibilistic approach (A) } \\
\text { (\% width reduction) }\end{array}$ & $\begin{array}{c}\text { Hybrid approach (B) } \\
\text { (\% width reduction) }\end{array}$ \\
\hline \multirow{3}{*}{$\boldsymbol{Z}_{\boldsymbol{c}}$} & $\boldsymbol{Z}_{\boldsymbol{c}}^{\mathbf{0 . 0 5}}$ & {$[50.70,51.67]$} & {$[50.90,51.56](0.3196)$} & {$[50.84,51.53](0.2887)$} \\
\cline { 2 - 5 } & $\boldsymbol{Z}_{c}^{\mathbf{0 . 5 0}}$ & {$[52.16,53.46]$} & {$[52.38,53.23](0.3462)$} & {$[52.36,53.28](0.2923)$} \\
\cline { 2 - 5 } & $\boldsymbol{Z}_{c}^{\mathbf{0 . 9 5}}$ & {$[54.13,56.44]$} & {$[54.21,55.87](0.2814)$} & {$[54.28,56.20](0.1688)$} \\
\cline { 2 - 5 } & $\boldsymbol{P}\left[\boldsymbol{Z}_{c}>\boldsymbol{z}_{c}{ }^{*}\right]$ & {$[0.8908,0.9946]$} & {$[0.9284,0.9921](0.3863)$} & {$[0.9103,0.9946](0.1879)$} \\
\hline
\end{tabular}

Table 5. Intervals $\left.\left[\mathrm{Pl}^{Z_{c}}\right]^{-1}(\beta),\left[\mathrm{Bel}^{Z_{c}}\right]^{-1}(\beta)\right]$ for the $\beta \cdot 100$-th percentiles $Z_{c}^{\beta}, \beta=0.05,0.50$ and 0.95, of the maximal water level of the river $Z_{c}$ (i.e., the model output) and intervals $\left[1-\bar{F}^{Z_{c}}\left(z_{c}^{*}\right), 1-\underline{F}^{Z_{c}}\left(z_{c}^{*}\right)\right]$ for the exceedance probability $P\left[Z_{c}>z_{c} *=55.5 \mathrm{~m}\right]$, before and after the Bayesian update performed by Approaches $A$ and $B$. The percentage reduction in the width of the intervals produced by Approaches $A$ and $B$ is also shown in parentheses for 
Approach A $\quad$ Approach B $\left(T=100, N_{\alpha}=21\right)$

Computational time $t_{\text {comp }}[\mathrm{s}]$ on a Intel® Core Co $2^{\mathrm{TM}}$ Duo CPUE7600@ 3.06 and $3.07 \mathrm{GHz}$

0.73

1536.65

1250 Table 6. Computational time $t_{\text {comp }}$ required by the Bayesian update of all the parameters of the PDFs of $Y_{1}=Q, Y_{2}=Z_{m}, Y_{3}=Z_{v}$ and $Y_{4}=K_{s}$ performed by Approach $A$ and by Approach $B$ with $T=100$ repetitions of the purely probabilistic Bayes' theorem for each of the $N_{\alpha}=21 \alpha$ - 
Purely possibilistic approach (A) Hybrid probabilistic-possibilistic approach

(B)

- High strength in updating the possibilistic

$\begin{array}{ll}\text { Advantages } & \begin{array}{l}\text { parameters independently on the size of the data } \\ \text { set available }\end{array} \\ \text { - Well-established and rigorous theoretical framework } \\ \text { (i.e., probabilistic Bayes' theorem) }\end{array}$

- Computationally cheap

- Necessity to "build" a possibilistic likelihood

- Necessity to "build" a (fictitious) Fuzzy Probability

$\begin{array}{ll}\text { Drawbacks } & \text { (e.g., by probability-possibility transformations, } \\ \text { normalization or directly from rough }\end{array}$ Distribution Functions as a prior

- High strength in updating the possibilistic parameters experimental data) only when the size of the data set available is large (e.g., > 100)

- Computationally burdensome

1256 Table 7. Advantages and drawbacks of the purely possibilistic $(A)$ and the hybrid probabilistic and 